Bazyli DEGÓRSKI O.S.P.P.E.*

\title{
ERETICI ED ERESIE NEL CHRONICON DI SAN GIROLAMO
}

Prima di comporre la sua opera più famosa, la Storia Ecclesiastica

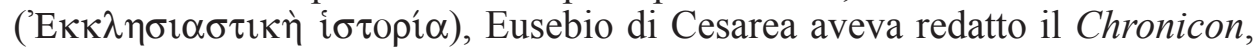
ossia un testo storiografico che riassume la cronologia universale e biblica. La versione originale greca dell'opera è andata persa, ma ne restano una traduzione completa in lingua armena ${ }^{1}$ e una parziale in latino, realizzata da san Girolamo ${ }^{2}$.

Dopo aver presentato una sintesi di storia orientale e greca in base alle fonti scritturistiche, nella seconda parte dell'opera Eusebio fornisce una tavola sincronica a più colonne che copre il periodo temporale da Abramo (2016 a.C.) sino all'anno 303 d.C., e proprio questa seconda parte del Chronicon eusebiano (noto anche con il titolo Temporum liber o Temporum breviarium) fu tradotta da san Girolamo e da lui stesso continuata sino all'anno 378 d.C. Il Dalmata lesse il Chronicon di Eusebio e lo tradusse nel 380 ca., durante il suo soggiorno a Costantinopoli ${ }^{3}$.

Nonostante numerosi errori e incongruenze, ripresi dal testo greco di Eusebio, ma anche commessi dallo stesso Girolamo, quest'opera storica è molto utile ancora oggi e, nel passato, ispirò il lavoro storiografico degli autori

${ }^{*}$ Prof. dr. hab. Bazyli Degórski, O.S.P.P.E. - professore di patrologia e di teologia dogmatica dell'età patristica presso la Pontificia Università S. Tommaso d'Aquino a Roma, e professore di patrologia e di antropologia patristica alla Pontificia Facoltà Teologica „Teresianum” a Roma; procuratore generale presso la Santa Sede; e-mail: osppe.roma@gmail.com.

${ }^{1}$ Cf. Eusebius Caesariensis, Chronicon bipartitum, nunc primum ex Armeniaco textu in Latinum conversum, adnotationibus auctum, Graecis fragmentis exornatum, ed. J.B. Aucher, Venetiis 1818 (versione armena e traduzione latina dall'armeno).

${ }^{2}$ Abbiamo le seguenti edizioni di quest'opera: Eusebius Caesariensis, Chronicon id est Temporum breviarium, ed. Ratdolt, Venetiis 1483; idem, Chronicorum libri duo, ed. A. Schoene, Berolini 1866-1875; idem, Werke, Bd. 5: Die Chronik, aus dem Armenischen übersetzt, hrsg. von J. Karst, GCS 20, Berlin 1911; idem, Werke, Bd. 7: Die Chronik des Hieronymus, hrsg. von R. Helm, 2. Aufl., GCS 47, Berlin 1956; [Girolamo], Hieronymi Opera 15 = Opere di Girolamo 15: Opere storiche e agiografiche, a cura di B. Degórski, Roma 2014, 209-307.

${ }^{3}$ Cf. J. Gribomont, Le traduzioni. Girolamo e Rufino, in: Quasten III 217. Secondo Albert Grisart (La Chronique de Saint Jérôme. Le lieu et la date de sa composition, „Hélikon” 2:1962, 248258), san Girolamo la scrisse a Treviri, negli anni 368-371! 
paleocristiani che la continuarono, quali ad esempio: Prospero di Aquitania ${ }^{4}$, Cassiodoro $^{5}$, o Vittore di Tunnuna ${ }^{6}$.

1. Donatisti. San Girolamo menziona nelle sue Cronache due volte Donato e i donatisti. La prima notizia è collocata all'anno 328 e presenta Donato come un personaggio ormai ben conosciuto e fondatore del donatismo diffuso nell'Africa latina: „Donato dunque, da cui si sviluppò il donatismo in Africa, è già ben noto" .

L'altra notizia risale all'anno 355 e parla dell'espulsione di Donato da Cartagine. Inoltre, san Girolamo ci fa sapere che i donatisti furono presenti anche a Roma e che alcuni di essi venivano chiamati „Montenses”, in quanto avevano nella Città Eterna una chiesa situata su una delle colline dell'Urbe. Quindi, quando Donato venne espulso da Cartagine, alcuni dei suoi seguaci furono denominati „Montenses” da un primo nucleo costituito da una chiesa su una collina a Roma ${ }^{8}$.

Recentemente, Matteo Dalvit ha presentato un'ipotesi che cerca di spiegare l'appellativo „Montenses” diversamente da ciò che afferma san Girolamo. Infatti, secondo Dalvit, quest'appellativo sarebbe derivato dall'esegesi di $\mathrm{Ab}$ 3,3 , fatta dai donatisti e basata sulla loro ecclesiologia scismatica, che identificava Donato con l'immagine di quel Santo (Sanctus) che arriva dal monte ombroso (mons umbrosus) di cui parla proprio il profeta Abacuc9.

2. Ariani. La prima notizia circa gli ariani, contenuta nelle Cronache di san Girolamo, risale all'anno 329 e riguarda la successione dei vescovi di Antiochia:

„Ad Antiochia, dopo Tiranno, viene ordinato $20^{\circ}$ vescovo Vital, dopo di lui Filogonio, al quale succedette $\left(22^{\circ}\right)$ Paolino, [e] dopo di lui $\left(23^{\circ}\right)$ Eustazio.

${ }^{4}$ Prosper Aquitanus, Epitoma Chronicae, PL 51, 535-606; ed. T. Mommsen, MGH Auctores Antiquissimi IX, Berolini 1892, 385-485; continuazione, ivi, 486-499; additamenta, PLS 3, 147148). Cf. anche A. Hamman, Scrittori della Gallia, in: Quasten III 525-526.

${ }^{5}$ Cassiodorus, Chronica, PL 69, 1213-1247; ed. T. Mommsen, MGH Auctores Antiquissimi XI, Berolini 1894, 109-161). Cf. anche U. Pizzani, Scrittori dell'Italia, in: Quasten IV 220.

${ }^{6}$ Victor Tunnunensis, Chronica, PL 68, 941-962; ed. T. Mommsen, MGH Auctores Antiquissimi XI, 163-206; ed. C. Cardelle de Hartmann, CCL 173A, Turnhout 2002. Cf. anche M. Simonetti, Letteratura cristiana d'Africa, in: Quasten IV 38-40; F. Scorza Barcellona, Vittore di Tunnuna, NDPAC III 5678-5679. Fra i ,continuatori” dell'opera geronimiana potrebbe essere annoverato forse anche Agapio di Gerapoli. Cf. Agapius Hierapolitanus, Gerapoli, Historia universalis, ed. B. Pirone: Agapio di Gerapoli Storia universale, introduzione, traduzione dall'arabo e note di B. Pirone, Studia Orientalia Christiana. Monographiae 21, Milano 2013.

${ }^{7}$ Hieronymus, «Chronicorum» Eusebii Caesariensis continuatio 276 Olymp., a. 328 post Christum natum, Hieronymi Opera 15, 248-249: „Donatus agnoscitur, a quo per Africam Donatiani”.

${ }^{8}$ Ibidem 283 Olymp., a. 355 post Christum natum, Hieronymi Opera 15, 274-275: „Donatus, a quo supra Donatianos in Africa dici memoravimus, Carthagine pellitur, quidam sectatores eius etiam Montenses vocant eo, quod ecclesiam Romae primum in monte habere coeperint".

${ }^{9}$ Cf. M. Dalvit, Montenses. The Ecclesiological Reflection of the Donatists Based on the Habacuc's Prophecy, „Augustiniana” 63 (2013) 173-188. 
Da quest'ultimo, costretto all'esilio a causa della [sua] fede, gli ariani presero la Chiesa di Antiochia tenendola fino al presente, cioè con Eulalio, Eusebio, Eufronio, Placillo, Stefano, Leonzio, Eudossio, Melezio, Euzoio, Doroteo e di nuovo Melezio. Non ho specificato le loro date, perché li ritengo nemici di Cristo piuttosto che vescovi" 10 .

Ora, vediamo più da vicino queste successioni episcopali ad Antiochia. Secondo la tradizione calcedonese, Tiranno fu vescovo di Antiochia dal 308 al 314. Secondo quella non calcedonese, invece, egli lo fu già dal 304 fin poi al $314^{11}$.

Per quanto concerne, Vitale, egli fu vescovo di Antiochia negli anni 314320 circa $^{12}$. Il successore di Vitale, san Filogonio, vescovo di Antiochia proprio all'inizio della stessa controversia ariana, fu avversario di Ario, e morì fra il 324 e il 325, poco tempo prima che cominciasse il primo Concilio ecumenico di Nicea del $325^{13}$.

Il successore di san Filogonio, Paolino I (o Paolino di Tiro), fu vescovo ariano di Antiochia, eletto nel 327 in luogo del deposto Eustazio; morì qualche mese dopo l'elezione ${ }^{14}$.

Al vescovo Paolino succedette ad Antiochia Eustazio. Egli era precedentemente vescovo di Berea (Aleppo) e poi proprio di Antiochia (324). Morì dopo il $343^{15}$.

Dopo Eustazio, secondo san Girolamo, cominciarono a presiedere la sede di Antiochia vescovi ariani. Primo di essi fu Eulalio, ma soltanto per qualche mese, in quanto morì nel $331^{16}$. Gli succedette, sempre secondo san Girolamo, Eusebio, ma questi è del tutto sconosciuto e non risulta in alcun elenco dei vescovi di Antiochia. Manlio Simonetti, invece, precisa che ad Eulalio succedette direttamente Eufronio ${ }^{17}$. Eufronio fu scelto proprio dagli ariani. Prima, però, essi avevano indicato per Antiochia Eusebio di Cesarea in Palestina, ma egli ricusò la proposta appellandosi al canone 15 del concilio di Nicea del 325 , che vietava le traslazioni dei vescovi da una sede ad un' altra. Allora, l'imperatore Costantino I propose il presbitero della Chiesa di Cesarea di Cappadocia,

${ }^{10}$ Hieronymus, «Chronicorum» Eusebii Caesariensis continuatio 277 Olymp., a. 329 post Christum natum, Hieronymi Opera 15, 248-251: „Antiochiae post Tyrannum XX ordinatur episcopus Vitalis, post quem XXI Filogonius, cui successit XXII Paulinus, post quem XXIII Eustathius, quo in exilium ob fidem truso usque in praesentem diem Ariani ecclesiam optinuerunt, id est Eulalius, Eusebius, Eufronius, Placillus, Stephanus, Leontius, Eudoxius, Meletius, Euzoius, Dorotheus, rursum Meletius, quorum idcirco tempora non digessi, quod eos hostes potius Christi quam episcopos iudicem".

${ }^{11}$ Cf. http://it.wikipedia.org/wiki/Patriarcato_di_Antiochia [consultato il 14 V 2016].

${ }^{12}$ Cf. S. Samulowitz, Vitale di Antiochia, NDPAC III 5664-5665.

${ }^{13}$ Cf. M. Simonetti, Filogonio di Antiochia, NDPAC II 1958.

${ }^{14}$ Cf. http://it.wikipedia.org/wiki/Patriarcato_di_Antiochia [consultato il 14 I 2013]; M. Simonetti, Paolino di Tiro, NDPAC III 3832.

${ }^{15}$ Cf. M. Simonetti, Eustazio di Antiochia, NDPAC I 1862-1863.

${ }^{16} \mathrm{Cf}$. idem, Eulalio di Antiochia, NDPAC I 1833.

${ }^{17} \mathrm{Cf}$. ibidem. 
Eufronio, e Giorgio di Aretusa. La scelta cadde su Eufronio, ma il suo governo durò soltanto un anno e pochi mesi; egli morì subito passato il $332^{18}$.

Dopo Eufronio, san Girolamo nomina Placillo, nome (Placillus) che gli è dato proprio da san Girolamo stesso. Si tratta piuttosto di Filaclo o Placenzio ${ }^{19}$.

A Placillo succedette Stefano. Si tratta di Stefano I, vescovo ariano di Antiochia, dal 342 al 344 circa, che fu deposto nel 344 a causa di uno scandalo ${ }^{20}$.

A Stefano I seguì, nel 344, uno dei discepoli di Luciano di Antiochia, Leonzio, di origine frigia. Seguendo l'insegnamento del maestro, Leonzio simpatizzò con le tesi di Ario. Fu perciò aspramente criticato da sant'Atanasio di Alessandria, anche a causa della sua castrazione e la coabitazione con la virgo subintroducta Eustolio. Leonzio nel 350 presiedette ad Antiochia un sinodo che stabilì di non comunicare con san'Atanasio di Alessandria, bensì soltanto con il vescovo ariano di quella città, Giorgio. Verso il 355, Leonzio ordinò diacono Aezio, capo degli ariani più rigidi e radicali, ma a motivo della protesta dei fedeli revocò Aezio dalla sua carica ecclesiastica. Leonzio morì nel 358 circa $^{21}$.

Segue il vescovo Eudossio, di origine armena. Egli appoggiò la propaganda ariana radicale proteggendo persino Aezio ed Eunomio. Nel 357 presiedette ad Antiochia un sinodo filoariano che approvò la formula sirmiese. In quel periodo, Eudossio dichiarò il Figlio dissimile dal Padre per sostanza e negò la sua reale generazione dal Padre. In seguito, però, si staccò dagli ariani radicali per posizioni più moderate e uscì trionfante dal sinodo di Costantinopoli del 360, tanto che fu trasferito al seggio episcopale di Costantinopoli, rimasto vacante dopo la deposizione di Macedonio. Resse Costantinopoli fino alla sua morte, avvenuta nel $370^{22}$.

Secondo le Cronache di san Girolamo, ad Eudossio succedette Melezio. Pare che egli sia stato vescovo di Antiochia nel 354. Da fonti calcedonesi sembra che sia riuscito, per breve tempo, a esautorare Eudossio ${ }^{23}$.

A Melezio succedette nel 360 Euzoio che in precedenza, già ai tempi di Ario, era stato diacono di Alessandria e molto collegato con lo stesso eresiarca. Il concilio di Nicea del 325 lo esiliò insieme ad Ario. Scrisse, in seguito all'imperatore Costantino una professione di fede per la quale il sinodo di Gerusalemme, del 335, lo riabilitò. Tuttavia, durante di sinodo di Seleucia, nel 359, si schierò con i filoariani. Eletto vescovo di Antiochia, nel 360, divenne il capo degli ariani moderati. Nel 361 conferì il battesimo all'imperatore Costanzo moribondo. Nel 362, sotto l'impero di Giuliano l'Apostata, celebrò ad Antiochia un sinodo di vescovi filoariani che cercò di riabilitare lo stesso

\footnotetext{
${ }^{18}$ Cf. A. Pollastri, Eufronio di Antiochia, NDPAC I 1826.

${ }^{19}$ Cf. http://it.wikipedia.org/wiki/Patriarcato_di_Antiochia [consultato il 14 I 2013].

${ }^{20} \mathrm{Cf}$. ibidem.

${ }^{21}$ Cf. M. Simonetti, Leonzio di Antiochia, NDPAC II 2776.

${ }^{22}$ Cf. idem, Eudossio, NDPAC I 1823-1824.

${ }^{23}$ Cf. http://it.wikipedia.org/wiki/Patriarcato_di_Antiochia [consultato il 14 I 2013].
} 
Aezio, staccandosi però dagli ariani radicali. Fu vescovo della comunità ariana ad Antiochia sino alla sua morte, avvenuta nel $375 \mathrm{circa}^{24}$.

Dopo la morte di Euzoio, fu eletto vescovo della comunità ariana di Antiochia Doroteo, che tenne questa sede episcopale sino al 381, quando l'editto antiariano dell'imperatore Teodosio il Grande lo fece allontanare. Prima di diventare vescovo ariano di Antiochia, Doroteo, dal 365 circa, fu vescovo di Eraclea (Tracia) di tendenza ariana moderata ed appoggiava Demofilo di Costantinopoli. Abbandonata la sede di Antiochia, Doroteo ritornò in Tracia, ma in seguito andò a Costantinopoli ad occupare l'incarico dell'eletto Marino, ritenuto dalla sua fazione inadatto ${ }^{25}$.

Alla fine di questa lista, san Girolamo, tra i vescovi filoariani, elenca anche Melezio. È ovvio che tale suo giudizio severo è influenzato dalle vicissitudini dello scisma di Antiochia ove, oltre a Melezio, operava il vescovo luciferiano Paolino, capo della cosiddetta „piccola Chiesa di Antiochia”, il quale ordinò presbitero san Girolamo. Melezio, di origine armena ed amico di Acacio di Cesarea, nel 358 o nel 360, fu eletto vescovo di Sebaste alla deposizione del vescovo Eustazio, ma i cristiani del luogo, fedeli ad Eustazio, si opposero e Melezio andò a Beroea. Nel 359 fu presente al sinodo di Seleucia.

Quando Eudossio si trasferì a Costantinopoli, Melezio alla fine del 360 lo sostituì ad Antiochia, ma pochi mesi dopo, a causa della sua tendenza antiariana, fu esiliato in Armenia. Però, nel 362, rioccupò la sede cattolica di Antiochia opponendosi al vescovo ariano della città, Euzoio. La tendenza filoariana dell'imperatore Valente lo obbligò, ben due volte (365 e nel 369), ad essere esiliato. Morto nel 378 Valente, Melezio ritornò dall'esilio e fu appoggiato addirittura da san Basilio Magno. Presiedette il secondo concilio ecumenico del 381, riunitosi a Costantinopoli, e morì durante i lavori di quest'assemblea ${ }^{26}$.

„Costantino, battezzato alla fine della sua vita da Eusebio, vescovo di Nicomedia, aderisce alla dottrina di Ario e da quel momento fino al tempo presente seguono attacchi alle Chiese e discordia in tutto il mondo"27, dichiara san Girolamo.

Eusebio fu dapprima vescovo di Berytus (l'odierna Beirut) in Fenicia e, in seguito, vescovo di Nicomedia e, infine, di Costantinopoli. Era imparentato con l'imperatore Costantino $\mathrm{I}^{28}$. In quanto simpatizzante con Ario, fu condannato

${ }^{24}$ Cf. M. Simonetti, Euzoio di Antiochia, NDPAC I 1876-1877.

${ }^{25}$ A proposito di Doroteo, cf. Socrates, HE IV 35, 4, ed. G.Ch. Hansen, GCS NF 1, Berlin 1995, 270, 21-24; V 23, 4-6, GCS NF 1, 305, 22 - 306, 3; Sozomenus, HE VII 17, 9-11, ed. J. Bidez - G.Ch. Hansen, GCS 50, Berlin 1960, 326, 8-18. Cf. anche M. Simonetti, Doroteo di Antiochia, NDPAC I 1508.

${ }^{26}$ Cf. M. Simonetti, Melezio di Antiochia, NDPAC II 3190-3191.

${ }^{27}$ Hieronymus, "Chronicorum» Eusebii Caesariensis continuatio 279 Olymp., a. 337 post Christum natum, Hieronymi Opera 15, 256-257: „Constantinus extremo vitae suae tempore ab Eusebio Nicomediensi episcopo baptizatus in Arianum dogma declinat. A quo usque in praesens tempus ecclesiarum rapinae et totius orbis est secuta discordia".

${ }^{28}$ Cf. Ammianus Marcellinus, Res gestae XXII 9, 4, ed. W. Seyfarth, I, Leipzig 1978, 271, 9-14. 
dal primo concilio di Nicea, nel 325, ed esiliato. Ciò nonostante, nel 328, venne richiamato dall'esilio e, nel 337, battezzò in punto di morte proprio Costantino I. Eusebio diventò vescovo di Costantinopoli nel 339. Morì nel $341 \mathrm{circa}^{29}$.

3. Giudizio di san Girolamo. Come possiamo costatare, san Girolamo vede nell'imperatore Costantino I un seguace dell'eresia ariana e, in tal modo, diviene l'iniziatore di una serie di quegli imperatori filoariani che perseguiteranno gli ortodossi praticamente fino all'arrivo dell'imperatore cattolico Teodosio I.

„La crudeltà degli ariani, sostenuta dall'imperatore Costanzo con l'utilizzo dell'esilio, della prigionia e di diversi tipi di tortura, perseguitò dapprima Atanasio e, successivamente, ogni vescovo che non fosse dalla loro parte" ${ }^{30}$.

Questo testo geronimiano, collocato all'anno 339, è molto chiaro e presenta l'imperatore in una luce piuttosto negativa. Si tratta di Costanzo II (Flavius Iulius Constantius), uno dei tre figli ed erede di Costantino I. Alla morte del padre nel 337, ottenne la prefettura d'Oriente e la diocesi di Tracia. Fu molto favorevole all'eresia ariana. Governò su tutto l'impero dal 350 al 361, anno in cui morì mentre si apprestava ad attaccare il suo rivale, Giuliano, detto in seguito l'Apostata, proclamato Augusto dall'esercito nel $360^{31}$.

„Ermogene, comandante dei soldati, viene linciato dalla folla di Costantinopoli poiché, sostenuto dall'imperatore e dalla fazione degli ariani, voleva bandire il vescovo Paolo" ${ }^{\prime 2}$.

Questa notizia geronimiana è collocata all'anno 342. Non sappiamo nient'altro di tale Ermogene, comandante dei soldati con l'appoggio dell'imperatore filoariano. Paolo, invece, fu eletto vescovo di Costantinopoli nel 332, anche se la sua nomina fu vista male dai vescovi limitrofi ${ }^{33}$. Sebbene che dalla notizia di san Girolamo Paolo potrebbe essere considerato un ortodosso zelante, egli prese parte, nel 335, al sinodo di Tiro firmando la condanna di sant'Atanasio di Alessandria, benché, a dir il vero, anch'egli, poco dopo fosse stato esiliato nel Ponto. Morto nel 337 l'imperatore Costantino I, Paolo tornò a Costantinopoli, ma fu di nuovo espulso e il suo posto venne occupato da Eusebio di Nicomedia

\footnotetext{
${ }^{29}$ Cf. Ch. Kannengiesser, Eusebio di Nicomedia, NDPAC I 1857-1860.

${ }^{30}$ Hieronymus, «Chronicorum» Eusebii Caesariensis continuatio 279 Olymp., a. 339 post Christum natum, Hieronymi Opera 15, 258-259: „Ex hoc loco impietas Ariana Constantii regis fulta praesidio exiliis carceribus et variis adflictionum modis primum Athanasium, deinde omnes non suae partis episcopos persecuta est".

${ }^{31}$ Cf. G. Gigli, L'ortodossia, l'arianesimo e la politica di Costanzo II, Napoli 1949; P.O. Cuneo, La legislazione di Costantino II, Costanzo II e Costante (337-361), Milano 1997; S. Laconi, Costanzo II. Ritratto di un imperatore eretico, Roma 2004; M. Forlin Patrucco, Costanzo II, NDPAC I 1247-1248.

${ }^{32}$ Hieronymus, "Chronicorum» Eusebii Caesariensis continuatio 280 Olymp., a. 342 post Christum natum, Hieronymi Opera 15, 260-261: „Hermogenes magister militiae Constantinopoli tractus a populo ob episcopum Paulum, quem regis imperio et Arianorum factione pellebat".

${ }^{33}$ Cf. Socrates, HE II 6, 7, GCS NF 1, 96, 23 - 97, 1; Sozomenus, HE III 3, 1-5, GCS 50, 104, 3-28.
} 
(un ariano dichiarato). Paolo si rifugiò a Roma e, morto proprio nel 342 circa il vescovo Eusebio, rientrò a Costantinopoli, ma i sostenitori filoariani di quest'ultimo contrapposero Macedonio a Paolo. La situazione allora divenne molto tesa, suscitando dei violenti tumulti popolari, a causa dei quali Paolo fu esiliato dapprima a Singara e in seguito ad Emesa. Con molta probabilità, quindi, san Girolamo accenna qui proprio a questa dolorosa situazione. Nel 344, Paolo ritornò a Costantinopoli, sostenuto sempre dal popolo, ma fu trasferito di nascosto a Tessalonica, amministrata non da Costanzo, bensì da Costante, e sotto la pressione del quale Costanzo II nel 346 permise a Paolo di ritornare a Costantinopoli. Quando, però, il sostenitore di Paolo, Costante, fu ucciso da Magnenzio e Costanzo cominciò a combattere contro di lui, nel 350 Paolo fu arrestato e trasferito a Cucusa, ove morì poco tempo dopo ${ }^{34}$.

\section{Macedoniani.}

„Macedonio, di mestiere fabbricante di broccati, sostituisce Paolo come vescovo degli ariani; con lui inizia l'eresia macedoniana" 35 .

„Macedonio viene bandito da Costantinopoli" ${ }^{\prime 36}$.

Queste notizie geronimiane sono collegate naturalmente con la precedente riguardante le vicissitudini di Paolo di Costantinopoli. Macedonio I fu eletto vescovo di Costantinopoli dopo l'esilio del vescovo ortodosso Paolo I, negli anni 342-348. Ritornato Paolo I, Macedonio abdicò, ma dopo la morte di Paolo, nel 350/351 circa, riprese la cattedra di Costantinopoli e la tenne sino al 360 , quando venne esiliato dagli ariani fondamentalisti ${ }^{37}$.

„La crudeltà del prefetto Filippo, sostenitore della fazione di Macedonio, e il complotto degli ariani portarono alla uccisione di Paolo"38.

Flavio Filippo (Flavius Philippus, 344-351), console e ufficiale sotto Costanzo II, era figlio di un macellaio e divenne un notarius $^{39}$. Nel 344 Filippo fu nominato da Costanzo II prefetto del pretorio d'Oriente; ottenne anche il

${ }^{34}$ Cf. M. Simonetti, Paolo di Costantinopoli, NDPAC III 3852-3853.

${ }^{35}$ Hieronymus, «Chronicorum» Eusebii Caesariensis continuatio 280 Olymp., a. 342 post Christum natum, Hieronymi Opera 15, 260-261: „Macedonius artis plumariae in locum Pauli ab Arianis episcopus subrogatur, a quo nunc haeresis Macedoniana".

${ }^{36}$ Ibidem 284 Olymp., a. 359 post Christum natum, Hieronymi Opera 15, 280-281: „Macedonius Constantinopoli pellitur".

${ }^{37}$ Circa Macedonio I, cf. Socrates, HE II 7, 1-2, GCS NF 1, 97, 1-4; II 13, 1-7, GCS NF 1, 104, 3 - 105, 5; II 16, 1-15, GCS NF 1, 107, 11 - 109, 9; II 38, 3-44, GCS NF 1, 164, 2 - 168, 22; II 40, 3-5, GCS NF 1, 171, 18-24; M. Simonetti, Macedonio (macedoniani), NDPAC II 2961-2962; cf. anche il lemma Macedoniusz I, SWP 270.

${ }^{38}$ Hieronymus, «Chronicorum» Eusebii Caesariensis continuatio 280 Olymp., a. 342 post Christum natum, Hieronymi Opera 15, 260-261: „Paulus crudelitate praefecti Philippi - nam fautor Macedonii partium erat - et Arianorum insidiis strangulatur".

${ }^{39}$ Libanius, Oratio 42, 24-25. 
consolato per l'anno 348. La sua politica religiosa seguì quella di Costanzo II, favorevole all'arianesimo. Proprio Flavio Filippo trasferì a Tessalonica il vescovo niceno di Costantinopoli, Paolo I, e lo sostituì con il vescovo ariano Macedonio I. Nel 351, per ordine di Filippo, Paolo I fu giustiziato a Cucusa, in Armenia ${ }^{40}$. Per quanto riguarda, invece, questa uccisione, per altri martirio, di Paolo I, abbiamo la seguente affermazione di Manlio Simonetti: „La notizia di fonte antica che lo vuole strangolato non sembra molto attendibile"41.

Massimino, vescovo di Treviri, viene considerato importante; Atanasio, vescovo di Alessandra fu da lui ricevuto con onore quando dovette scontare la punizione inflittagli da $\operatorname{Costanzo}^{42}$.

5. Ripristino dell'ortodossia. Girolamo stabilisce che „Atanasio fa ritorno ad Alessandria in seguito ad una lettera di Costanzo"43. Lo appoggia Massimino (Maximinus, 330-346/347, vescovo di Treviri al tempo dei figli di Costantino il Grande, uno dei maggiori avversari di Ario). Infatti, a sant'Atanasio Massimino diede asilo a Treviri dal 335 al $337^{44}$.

Il 26 giugno 345 morì il vescovo ariano di Alessandria Gregorio. Quindi, Costanzo acconsentì alle ripetute richieste di Costante, affinché ritornassero alle loro sedi i vescovi niceni esiliati. Costante fece venire sant'Atanasio a Treviri e gli comunicò la notizia. In seguito, a Roma papa Giulio gli consegnò proprio la lettera secondo la quale doveva rioccupare la sede vescovile di Alessandria. Poi Atanasio si incontrò con Costanzo ad Antiochia e quest'ultimo scrisse una lettera ai fedeli di Alessandria raccomandando loro di vivere in pace. Facendo ritorno alla sua sede, sant'Atanasio attraversò la Siria, la Fenicia, la Palestina e, infine, il 21 ottobre 346 fu trionfalmente accolto dai fedeli di Alessandria ${ }^{45}$.

„Eusebio di Emesa, capo della fazione ariana, compone molte e diverse opere" ${ }^{\text {. }}$.

${ }^{40}$ Circa Flavio Filippo, cf. A.H.M. Jones, The Career of Flavius Philippus, „Historia” 4 (1955) 229-233; L.J. Swift - H.O. James, Constantius II on Flavius Philippus, „The American Journal of Philology" 83 (1962) 247-264; J. Boardman, The Cambridge Ancient History, Cambridge 1970, 20, 171 e 353; The Prosopography of the Later Roman Empire, ed. A.H.M Jones - J.R. Martindale - J. Morris, III, Cambridge 1992, 696-697.

${ }^{41}$ Simonetti, Paolo di Costantinopoli, NDPAC III 3853.

${ }^{42}$ Cf. Hieronymus, «Chronicorum» Eusebii Caesariensis continuatio 280 Olymp., a. 343 post Christum natum, Hieronymi Opera 15, 260-261: „Maximinus Treverorum episcopus clarus habetur, a quo Athanasius Alexandriae episcopus, cum a Constantio quaereretur ad poenam, honorifice susceptus est".

${ }^{43}$ Ibidem 281 Olymp., a. 346 post Christum natum, Hieronymi Opera 15, 262-263: „Athanasius ad Constantis litteras Alexandriam regreditur".

${ }^{44} \mathrm{Cf}$. ibidem 280 Olymp., a. 343 post Christum natum, Hieronymi Opera 15, 262: „Maximinus Treverorum episcopus clarus habetur, a quo Athanasius Alexandriae episcopus, cum a Constantio quaereretur ad poenam, honorifice susceptus est".

${ }^{45}$ Cf. A. Camplani, Atanasio di Alessandria, NDPAC I 622.

${ }^{46}$ Hieronymus, "Chronicorum» Eusebii Caesariensis continuatio 281 Olymp., a. 347 post 
Questa notizia di san Girolamo risale all'anno 347: il Dalmata è il primo a fornirla. Purtroppo, san Girolamo presenta Eusebio addirittura come „capo della fazione ariana"! Eusebio vescovo di Emesa, in Fenicia, era nato ad Edessa nel 300 circa, era siro di stirpe ma greco di formazione e morì ad Antiochia nel 359 circa. Fu uno dei discepoli di Eusebio di Cesarea e perfezionò la sua preparazione ad Antiochia e Alessandria ${ }^{47}$. Forse per tale motivo, Eusebio viene considerato filoariano, cioè per il suo legame stretto con Eusebio di Cesarea in Palestina.

Per quanto riguarda le opere di Eusebio di Emesa, così le presenta lo stesso san Girolamo nel De viris inlustribus:

„Tra le sue opere, particolarmente significativi sono i trattati Contro i Giudei, i pagani e i seguaci di Novaziano, i dieci libri Sulla lettera ai Galati ${ }^{48}$ e le brevi ma numerosissime Omelie sui Vangeli ${ }^{49}{ }^{, 50}$.

\section{Ancora lotte altalenanti per la verità.}

„Muore Massimo, il $40^{\circ}$ vescovo di Gerusalemme dopo Macario; dopo di lui gli ariani prendono possesso della Chiesa con Cirillo, Eutichio, ancora Cirillo, Ireneo, Cirillo una terza volta, Ilario, Cirillo una quarta volta. Di questi, Cirillo era stato ordinato presbitero da Massimo e, quindi, dopo la sua morte l'episcopato gli fu promesso da Acacio, vescovo di Cesarea ${ }^{51}$, e da altri ariani, se egli avesse ripudiato l'ordinazione da parte di Massimo. Egli servì come diacono

Christum natum, Hieronymi Opera 15, 264-265: „Eusebius Emisenus Arianae signifer factionis multa et varia conscribit".

${ }^{47}$ Cf. S.G. Krüger, Eusebius, Bischof von Emesa (Emisa) in Phönizien, in: Realencyklopädie für protestantische Theologie und Kirche, V, Leipzig 1898, 618-619; M. Simonetti, Eusebio di Emesa, NDPAC I 1854-1855. Così presenta Eusebio di Emesa lo stesso san Girolamo nel De viris inlustribus 91, 1 e 3, Hieronymi Opera 15, 423: „Eusebio di Emesa, di ingegno brillante ed esperto nella retorica, compose innumerevoli opere, tali da suscitare l'applauso popolare, avvalendosi particolarmente di un'esegesi letterale; viene letto con moltissima attenzione da coloro che vogliono parlare in pubblico. [...]. Fiorì al tempo dell'imperatore Costanzo, sotto il quale morì e fu sepolto ad Antiochia” („Eusebius Emisenus, elegantis et rhetorici ingenii, innumerabiles et qui ad plausum populi pertineant confecit libros, magisque historiam secutus, ab his, qui declamare volunt, studiosissime legitur; [...]. Floruit temporibus Constantii imperatoris, sub quo et mortuus Antiochiae sepultus est" - ibidem, p. 422).

${ }^{48}$ Di tali opere sono pervenuti soltanto frammenti di carattere esegetico.

${ }^{49}$ Pervenuto in traduzione latina un gruppo di 29 omelie, quasi tutte concernenti aspetti dottrinali; l'esegesi è a tendenza fortemente letteralista.

${ }^{50}$ Hieronymus, De viris inlustribus 91, 2, Hieronymi Opera 15, 422-423: „, quibus vel praecipui sunt Adversum Iudaeos et gentes et Novatianos et Ad Galatas libri decem, et In Evangelia homiliae breves, sed plurimae".

${ }^{51}$ Acacio di Cesarea ('Aкókııৎ [chiamato dai suoi avversari „il guercio" - Movó $\varphi \theta \alpha \lambda \mu \circ \varsigma$ ]; ?-365 circa) fu vescovo ariano, discepolo e biografo di Eusebio di Cesarea, del quale fu successore, circa dal 340/341. A proposito di lui, cf. J.-M. Leroux, Acace, évêque de Césarée de Palestine (341365), StPatr 8 (1966) 82-85; M. Simonetti, Acacio di Cesarea, NDPAC I 36-37. 
nella Chiesa. Gli venne offerta una ricompensa a riparazione dell'azione empia che aveva subito. Egli degradò da vescovo a presbitero Eraclio, che era stato nominato vescovo al suo posto da Massimo in punto di morte, tormentandolo con vari imbrogli’"52.

Questa notizia geronimiana è collocata all'anno 348 e riguarda prevalentemente san Cirillo di Gerusalemme. Macario I fu vescovo di Gerusalemme dal 312 circa a poco prima del 335, e fu uno dei più accaniti avversari di Ario al primo concilio di Nicea nel $325^{53}$. Massimo III, invece, fu vescovo di Gerusalemme negli anni 333-348 ca. ${ }^{54}$. San Cirillo nacque a Gerusalemme nel 313 o 315 e morì, sempre a Gerusalemme, il 18 marzo 387. In qualità di presbitero di Gerusalemme ordinato dal niceno Massimo, proprio nel $348 \mathrm{fu}$ ordinato vescovo dagli eusebiani Patrofilo di Scitopoli e Acacio di Cesarea in Palestina, e ciò in luogo del presbitero Eraclio. Entrato in disaccordo dottrinale e giurisdizionale con lo stesso Acacio, nel 357 fu deposto durante un sinodo di Gerusalemme. Quindi gli eusebiani si divisero; Cirillo optò per gli omeousiani i quali nel 359 lo riabilitarono durante il sinodo di Seleucia. Tuttavia, nel 360, il sinodo di Costantinopoli, dominato da Acacio e dagli omei, lo depose di nuovo. Dopo la morte di Costanzo, nel 362, ritornò alla propria sede episcopale, ma a causa della linea filoariana di Valente, fu nuovamente esiliato o nel 367 o qualche anno dopo. Nel 378 ritornò a Gerusalemme e partecipò al concilio di Costantinopoli del 381 e ivi ancora al sinodo del 382. In quest'ultima assise venne riconosciuta la validità della sua ordinazione vescovile ${ }^{55}$. Seguono due notizie geronimiane riguardanti papa Liberio:

„Liberio viene ordinato $34^{\circ}$ vescovo della Chiesa romana; quando fu costretto all'esilio per motivi di fede, tutto il clero giurò che non avrebbe accettato alcun altro. Ma quando Felice fu istituito vescovo dagli ariani, molti spergiurarono e, dopo un anno, furono cacciati con Felice, perché Liberio, annoiato dal tedio dell'esilio e appoggiando la depravazione eretica, aveva fatto ingresso a Roma come un vincitore" 56 .

${ }^{52}$ Hieronymus, «Chronicorum» Eusebii Caesariensis continuatio 281 Olymp., a. 348 post Christum natum, Hieronymi Opera 15, 264-265: „XL Maximus post Macarium Hierosolymarum episcopus moritur, post quem ecclesiam Ariani invadunt, id est Cyrillus, Eutychius, rursum Cyrillus, Irenaeus, tertio Cyrillus, Hilarius, quarto Cyrillus, quorum Cyrillus, cum a Maximo fuisset presbyter ordinatus et post mortem eius ita ei ab Acacio episcopo Caesariensi et ceteris Arianis episcopatus promitteretur, si ordinationem Maximi repudiasset, diaconus in ecclesia ministravit, ob quam impietatem sacerdotii mercede pensatus Heraclium, quem moriens Maximus in suum locum substituerat, varia fraude sollicitans de episcopo in presbyterum regradavit".

${ }^{53}$ Cf. O. Zöckler, Macarius, in: New Schaff-Herzog Encyclopedia of Religious Knowledge, ed.

S.M. Jackson, VII, London - New York 1914³, 103; S.J. Voicu, Macario I, NDPAC II 2953.

${ }^{54}$ Cf. J.-M. Sauget, Massimo, NDPAC II 3116-3118.

${ }^{55}$ Cf. M. Simonetti, Cirillo di Gerusalemme, NDPAC I 1050-1052; M. Starowieyski, Cirillo di Gerusalemme (Pseudo), NDPAC I 1052.

${ }^{56}$ Hieronymus, "Chronicorum» Eusebii Caesariensis continuatio 282 Olymp., a. 349 post 
Papa Liberio fu vescovo di Roma dal 17 maggio 352 alla sua morte (avvenuta il 24 IX 366$)^{57}$. Felice, invece, fu vescovo romano che divenne antipapa con il nome di Felice II, dal 356 alla sua morte (il 22 XI 365, in Portus Romae, Fiumicino $)^{58}$. Papa Liberio fece celebrare un sinodo a Roma per regolare la questione di sant'Atanasio, ma il sinodo fallì. Si rivolse, perciò, a Costanzo affinché facesse riunire un concilio ad Aquileia. Costanzo, invece, fece condannare sant'Atanasio ben due volte: dal sinodo di Arles (353) e da quello di Milano (355). Liberio, però, non accettò tale sentenza e Costanzo lo esiliò, nel 357, in Tracia ${ }^{59}$. San Girolamo colloca questo esilio all'anno 356: „Liberio, vescovo romano, viene mandato in esilio"60.

Costanzo II ordinò al praefectus urbis di Roma, Flavio Leonzio, di arrestare papa Liberio. L'imperatore esiliò Liberio a Beroea in Tracia. Al suo posto, Costanzo II fece eleggere l'arcidiacono Felice ${ }^{61}$. Il papa maltrattato e debole durante l'esilio accettò la volontà dell'imperatore e Costanzo II permise a Liberio di ritornare dapprima a Sirmio (358) e, quindi, a Roma, dove fu accolto benevolmente dai fedeli, obbligando Felice II ad andarsene. Sotto l'impero di Giuliano l'Apostata (361-363) Liberio, in una epistola indirizzata ai vescovi d'Italia (362363 ) e in una in risposta ai vescovi orientali (del 366), si dichiaro palesemente favorevole al Credo Niceno del $325^{62}$. La seguente notizia risale all'anno 354:

„I vescovi delle province galliche Paolino e Rodanio sono costretti all'esilio per la loro fede" $"$.

Di Paolino di Treviri san Girolamo afferma anche (all'anno 358): „Paolino, vescovo di Treviri, muore in esilio in Frigia" 64 .

Vescovo di Treviri, Paolino (Paulinus) fu ordinato presbitero dal già citato Massimino di Treviri, e verso il 347 fu ordinato vescovo di Treviri. Durante la

Christum natum, Hieronymi Opera 15, 266-267: „Romanae ecclesiae XXXIIII ordinatur episcopus Liberius, quo in exilium ob fidem truso omnes clerici iuraverunt, ut nullum alium susciperent. Verum cum Felix ab Arianis fuisset in sacerdotium substitutus, plurimi peieraverunt et post annum cum Felice eiecti sunt, quia Liberius taedio victus exilii et in haeretica pravitate subscribens Romam quasi victor intraverat".

${ }^{57}$ Cf. LP I 207-210; B. Studer, Liberio papa, NDPAC II 2822-2823.

${ }^{58} \mathrm{Cf}$. Gesta inter Liberium et Felicem episcopos 1, ed. O. Guenther, in: Collectio Avellana, CSEL 35/1, Pragae - Vindobonae - Lipsiae 1895, 1-4; P. Marone, Felice II antipapa, NDPAC II 1927.

${ }^{59} \mathrm{Cf}$. le quattro lettere di Liberio conservate in: Hilarius Pictaviensis, Fragmenta historica 4, 6. Cf. anche Studer, Liberio papa, NDPAC II 2822-2823.

${ }^{60}$ Hieronymus, «Chronicorum» Eusebii Caesariensis continuatio 283 Olymp., a. 356 post Christum natum, Hieronymi Opera 15, 278-279: „Liberius episcopus Romanus in exilium mittitur”.

${ }^{61}$ Cf. Marone, Felice II antipapa, NDPAC II 1927; Studer, Liberio papa, NDPAC II 2822-2823.

${ }^{62}$ Cf. Studer, Liberio papa, NDPAC II 2822-2823.

${ }^{63}$ Hieronymus, «Chronicorum» Eusebii Caesariensis continuatio 283 Olymp., a. 354 post Christum natum, Hieronymi Opera 15, 272-273: „Paulinus et Rodanius Galliarum episcopi in exilium ob fidem trusi”.

${ }^{64}$ Ibidem 284 Olymp., a. 358 post Christum natum, Hieronymi Opera 15, 278-279: „Paulinus Trevirorum episcopus in Frygia exulans moritur". 
disputa tra Ario e sant'Atanasio, Paolino appoggiò sant'Atanasio, che aveva trovato asilo proprio a Treviri. Nell'anno 353, Costanzo II convocò il sinodo ad Arles, durante il quale i seguaci di Ario presentarono un progetto dell'imperatore che condannava sant'Atanasio. Costanzo II, inoltre, emise un editto secondo cui i vescovi che non accettavano la condanna di Atanasio venivano minacciati di ostracismo. Tutti i vescovi presenti al sinodo di Arles sottoscrissero la proposta di Costanzo, tranne proprio il vescovo Paolino, che fu esiliato in Frigia, dove morì verso il $358^{65}$.

Rodanio (Rhodanius), invece, fu vescovo di Tolosa circa negli anni 350358. Le fonti storiche a nostra disposizione ci danno notizie divergenti riguardanti il sinodo che esiliò Rodanio. Come abbiamo visto, san Girolamo sembra pensare al sinodo di Arles del 353. Rufino di Aquileia, invece, e lo storico Sozomeno, parlando di un solo sinodo precedente al sinodo di Rimini, elencano cinque vescovi, tra cui anche Rodanio, condannati a Milano nel 355, ed aggiungono a loro Ilario di Poitiers, ma come se egli fosse stato mandato in esilio in un'altra data ${ }^{66}$. Sulpicio Severo, invece, parlando dei sinodi di Arles, Béziers e di Milano, non spiega quando Rodanio si oppose all'imperatore Costanzo II (forse dopo il sinodo di Milano), ma tratta di Rodanio assieme ad Ilario di Poitiers, constatando che il vescovo di Tolosa doveva all'esempio di Ilario la forza e la costanza nell'opporsi all'imperatore. Inoltre, Sulpicio Severo ci informa che, sotto i consoli Arbizione e Lolliano, Ilario e Rodanio andarono in esilio e che Rodanio morì esiliato in Frigia ${ }^{67}$. Sant'Ilario di Poitiers, infine, le notizie del quale sono forse le più attendibili, vede nel sinodo di Béziers del 356 l'assemblea durante la quale Rodanio si oppose a Costanzo II ${ }^{68}$.

Gli ariani e Costanzo condannano all'esilio Eusebio, vescovo di Vercelli, Lucifero e Dionigi, vescovi delle Chiese di Cagliari e Milano, anche Pancrazio, presbitero romano ${ }^{69}$ ed Ilario, un diacono ${ }^{70}$, tutti lontani l'uno dall'altro ${ }^{71}$.

Sant'Eusebio di Vercelli (nato in Sardegna, circa nel 283 - morto a Vercelli, il $1^{\circ}$ agosto 371 ) fu il primo vescovo di Vercelli e uno dei principali antagonisti dell'eresia ariana. Fu inviato da papa Liberio, insieme al vescovo

${ }^{65}$ Cf. M. Simonetti, Paolino di Treviri, NDPAC III 3832-3833.

${ }^{66}$ Cf. Rufinus Aquileiensis, HE I 20, PL 21, 493A-B; Sozomenus, HE IV 9, 1-4, GCS 50, $148,11-24$.

${ }^{67}$ Cf. Sulpicius Severus, Chronica II 45, 9, ed. C. Halm, CSEL 1, Vindobonae 1866, 99, 11-12.

${ }^{68}$ Cf. Hilarius Pictaviensis, Fragmenta historica 6, 1-2; idem, Contra Constantium Augustum 2, ed. A. Rocher, SCh 334, Paris 1987, 170, 11-14. A proposito di Rodanio, cf. H. Crouzel - L. Odrobina, Rodanio di Tolosa, NDPAC III 4573-4574.

${ }^{69} \mathrm{Cf}$. G. Corti, Lucifero di Cagliari. Una voce nel conflitto tra chiesa e impero alla metà del IV secolo, SPM 24, Milano 2004, 50, 52, 61, 71, 171 e 180.

${ }^{70} \mathrm{Cf}$. ibidem, p. 50 e passim.

${ }^{71} \mathrm{Cf}$. Hieronymus, «Chronicorum» Eusebii Caesariensis continuatio 283 Olymp., a. 355 post Christum natum, Hieronymi Opera 15, 274-275: „Eusebius Vercellensis episcopus et Lucifer ac Dionysius Calaritanae et Mediolanensis ecclesiae episcopi, Pancratius quoque Romanus presbyter et Hilarius diaconus distantibus inter se ab Arianis et Constantio damnantur exiliis". 
san Lucifero di Cagliari, in missione presso Costanzo II per domandargli la convocazione di un sinodo che ponesse fine alla controversia ariana. L'assemblea ebbe luogo a Milano nel 355 ma, essendo i vescovi ariani in maggioranza e appoggiati dallo stesso Costanzo II, Eusebio si rifiutò di sottoscriverne le decisioni. L'imperatore esiliò Eusebio a Scitopoli in Palestina prima, poi in Cappadocia e, quindi, nella Tebaide. Morto Costanzo II, nel 362 Giuliano gli consentì di riprendere la sua sede vescovile. Nel 362, Eusebio partecipò al Concilio di Alessandria che decise di perdonare i vescovi ariani ${ }^{72}$.

San Lucifero (nato probabilmente a Cagliari, III secolo - morto a Cagliari, nel 370) fu vescovo di Cagliari. Combatté contro Ario e si oppose alla condanna di sant'Atanasio al Concilio di Milano del 355. Venne condannato da Costanzo II all'esilio in Siria, poi in Palestina e, infine, in Egitto. Giuliano promulgò un editto (362) che permetteva ai vescovi esiliati di rientrare nelle loro sedi. Nello stesso anno, sant'Atanasio convocò un sinodo ad Alessandria per porre fine alle dispute dogmatiche, ed invitò anche Lucifero, il quale però andò ad Antiochia, dove ordinò vescovo Paolino, il quale appoggiava Eustazio, uno dei difensori dell'ortodossia nicena. Rientrato a Cagliari, Lucifero si oppose alle decisioni del Concilio di Alessandria di reintegrare i vescovi ariani. Le sue idee gli attirarono molti seguaci, iniziando in tal modo il cosiddetto scisma luciferiano ${ }^{73}$.

Dionigi (nato? - morto in Cappadocia, 360) fu vescovo di Milano dal 349 circa (succedendo ad Eustorgio I) alla sua deposizione nel 355. Partecipò nel 355 al Concilio di Milano riunitosi allo scopo di deporre sant'Atanasio. Essendo Dionigi in ottimi rapporti con Costanzo II, sottoscrisse la condanna di Atanasio. Tuttavia, quando arrivò da Vercelli il vescovo Eusebio che, invalidando la condanna per vizio di forma, impose ai vescovi presenti la professione di fede nicena, Costanzo II ordinò che venisse rinnovata la sentenza di condanna. Sapendo che questa sentenza non rispondeva alle decisioni del Concilio di Nicea (325), Dionigi di Milano, Eusebio di Vercelli e Lucifero di Cagliari si rifiutarono di eseguirla e, quindi, furono esiliati. Dionigi fu esiliato in Cappadocia e la sede vescovile di Milano fu occupata dal vescovo ariano Aussenzio ${ }^{74}$.

„Ilario, vescovo di Poitiers, esiliato prima del triennio in Frigia in seguito ad un complotto di Saturnino, vescovo di Arles, e degli altri ariani a lui vicini, scrive libri sulla nostra religione" $"$.

${ }^{72}$ Cf. A. Jülicher, Eusebios 27, RE VI/1, 1441-1443; F.W. Bautz, Eusebius von Vercelli, BBKL I 1568-1569; N. Everett, Narrating the Life of Eusebius of Vercelli, in: Narrative and History in the Early Medieval West, ed. R. Balzaretti - E.M. Tyler, Turnhout 2006, 133-165; L. Dattrino, Eusebio di Vercelli, NDPAC I 1861-1862.

${ }^{73}$ Cf. E. Sauser, Lucifer, Bischof von Calaris, BBKL V 298-301; Corti, Lucifero di Cagliari, passim; M. Simonetti, Lucifero (luciferiani), NDPAC II 2935-2937.

${ }^{74}$ Cf. M. Simonetti, Dionigi di Milano, NDPAC I 1443.

${ }^{75}$ Hieronymus, "Chronicorum» Eusebii Caesariensis continuatio 283 Olymp., a. 356 post Christum natum, Hieronymi Opera 15, 276-277: „Hilarius episcopus Pictaviensis factione Saturnini Arelatensis episcopi reliquorumque, qui cum eo erant, Arianorum ante triennium in Frygiam 
Sant'Ilario di Poitiers (nato a Poitiers, nel 315 circa - morto a Poitiers, nel 367) fu vescovo di Pictavium (l'odierna Poitiers in Francia). Nel 356, il sinodo di Béziers, convocato dai vescovi filoariani, depose sant'Ilario a motivo della sua separazione dalla comunione con Valente, Ursacio e Saturnino di Arles, e sant'Ilario venne esiliato in Frigia da Costanzo II $^{76}$.

Infine, Saturnino vescovo ariano di Arles (347?-360 o 361) appoggiò la politica ariana di Costanzo II e fece condannare (356) sant'Ilario di Poitiers. Saturnino fu deposto dal Concilio di Parigi (360 o 361$)^{77}$.

Vengono convocati i sinodi di Rimini e di Seleucia in Isauria, nei quali viene condannata l'antica fede dei padri, dapprima per il tradimento di dieci delegati, poi da parte di tutti i partecipanti ${ }^{78}$.

7. I Sinodi di Rimini e di Seleucia. Il sinodo di Rimini si aprì alla presenza di ca. 400 vescovi, verso il principio di giugno del 359, e fu indetto dall'imperatore Costanzo per la parte occidentale dell'impero per discutere le diverse posizioni teologiche (ortodosse e filoariane) emerse nel 357-358, mentre a Seleucia veniva celebrato, in parallelo, un altro sinodo desinato ai vescovi della parte orientale. Il sinodo di Rimini fu presieduto da Restituto di Cartagine. Papa Liberio fu assente. I vescovi della minoranza ariana, Valente di Mursa, Ursacio di Singidunum (l'odierna Belgrado) e Germinio di Sirmio,

pulsus libros de nostra religione componit". La più importante opera di sant'Ilario di Poitiers è il De Trinitate (ed. P. Smulders, t. 1-3, SCh 443, 448 e 462, Paris 1999-2000-2001). Le altre opere di Ilario sono le seguenti: Hymni (Hilarius Pictaviensis, Opera, ed. A.L. Feder, CSEL 65, Vindobonae 1916, 207-223; PLS 1, 271-281), ritrovati nel 1887, di argomento dogmatico; Fragmenta historica (CSEL 65, 39-193; PL 10, 627-724); Contra Arianos vel contra Auxentium Mediolanensem (PL 10, 609-618); Contra Constantium Augustum, ed. A. Rocher, SCh 334, Paris 1987, 166-223; PL 10, 578-604); Adversus Valentem et Ursacium, historiam Ariminensis et Seleuciensis synodi continens (l'opera per noi perduta); Commentarius in Evangelium Matthaei (ed. J. Doignon, t. 1-2, SCh 254 e 258, Paris 1978-2007; PL 9, 917-1076; ed. A.L. Feder in: Hilarius Pictaviensis, Opera, CSEL 65, 232 [frammenti]); Tractatus super Psalmos (ed. J. Doignon, SCh 515, Paris 2008; ed. J. Doignon - R. Demeulenaere, CCL 61-61A-B, Turnhout 1997-2009); De synodis seu de fide orientalium (PL 10, 471-546); Apologetica ad reprehensores libri De synodis responsa (PL 10, 545-548); De mysteriis (ed. A.L. Feder, CSEL 65, 1-38; PLS 1, 246-270; ed. J.-P. Brisson, SCh 19bis, Paris 1967).

${ }^{76} \mathrm{Cf}$. А. Орловъ, Тринитарныл воззрьнія Иларія Пиктавійскаго: историко-догматическое изсльдованіе, Сергіевъ Посадъ 1908; idem, Христологія Иларія Пиктавійскаго: въ связи съ обзоромъ христологическихъ ученій 2-4 вв., Сергіевъ Посадъ 1909; H. Lietzmann, Hilarius 11, RE VIII/2, 1601-1604; P. Smulders, La Doctrine trinitaire de St. Hilaire de Poitiers. Esquisse du mouvement dogmatique depuis le Concile de Nicée jusqu'au règne de Julien, Analecta Gregoriana 32, Roma 1944; B. Mondin, Dizionario dei Teologi, Bologna 1992, 308-313; M. Simonetti, Ilario di Poitiers, NDPAC III 2521-2528; Dieu Trinité d'hier à demain avec Hilaire de Poitiers, Actes du congrès-colloque du Futuroscope de Poitiers, nov. 2002, ed. D. Bertrand, Paris 2010.

${ }^{77}$ Cf. V. Saxer - S. Samulowitz, Saturnino di Arles, NDPAC III 4768-4769.

${ }^{78}$ Cf. Hieronymus, «Chronicorum» Eusebii Caesariensis continuatio 284 Olymp., a. 359 post Christum natum, Hieronymi Opera 15, 280-281: „Synhodus apud Ariminum et Seleuciam Isauriae facta, in qua antiqua patrum fides decem primum legatorum, dehinc omnium proditione damnata est". 
condannarono di nuovo lo ó del Credo filoariano presentato a Sirmio il 22 maggio 359. Ciò nonostante, la maggioranza dei partecipanti confermò il Credo niceno e il 21 luglio 359 Valente e i suoi sostenitori furono scomunicati. Quindi, un'ambasceria, guidata da Restituto andò a Costantinopoli dall'imperatore Costanzo.

Questi, però, non accettò lo ó a Nike, ove Valente costrinse gli occidentali a firmare un Credo secondo il quale il Figlio veniva presentato come simile al Padre secondo le Scritture. In seguito, la delegazione tornò a Rimini e gli occidentali, costretti e maltrattati, accettarono a poco a poco la cosiddetta formula di Rimini, ove il Figlio viene definito simile al Padre e i termini ó e banditi. La formula di Rimini, in quanto del tutto generica, poteva essere intesa anche secondo le tesi ariane. Il sinodo, perciò, fu visto come un'altra vittoria dei sostenitori di Ario $^{79}$.

Il parallelo sinodo di Seleucia d'Isauria (Asia Minore), invece, fu aperto il 27 settembre 359 e durò soltanto pochi giorni. Vi parteciparono circa 160 vescovi di maggioranza ariana presieduta da Eustazio di Sebaste, Eleusio di Cizico e Silvano di Tarso. Fu imposta la formula filoariana di Antiochia del 341 e bandita quella presentata da Acacio di Cesarea che non accettava né

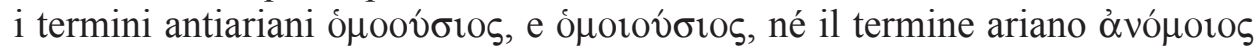
e considerava il Figlio simile al Padre. I risultati del sinodo furono comunicati in ottobre all'imperatore Costanzo il quale, favorendo la formula di Acacio, non vide positivamente questa vicenda.

Nel frattempo però, arrivò la notizia che i vescovo riuniti a Rimini accettarono, anche se forzosamente, la formula gradita all'imperatore. Allora, Costanzo fece sottoscrivere la formula di Rimini anche ai sinodali di Seleucia ${ }^{80}$.

\section{Origine dell'eresia pneumatomaca.}

„Ilario rientra nelle province galliche dopo che, a Costantinopoli, aveva offerto un libro in propria difesa a Costanzo" $"$.

Si tratta probabilmente del Contra Constantium Augustum ${ }^{82}$. Nel 359, sant'Ilario di Poitiers compose il Liber II ad Constantium. Finito il sinodo di Seleucia, Ilario andò a Costantinopoli e indirizzò a Costanzo una richiesta pregando l'imperatore di autorizzare una disputa con Saturnino di Arles che era stato il principale responsabile della condanna di Ilario al sinodo di Béziers. Tale sua richiesta non fu soddisfatta, quindi, Ilario compose un'opera intitolata

\footnotetext{
${ }^{79}$ Cf. M. Simonetti, Rimini (concilio), NDPAC III 4509-4511.

${ }^{80}$ Cf. idem, Seleucia d'Isauria (concilio), NDPAC III 4843-4844.

${ }^{81}$ Hieronymus, "Chronicorum» Eusebii Caesariensis continuatio 284 Olymp., a. 359 post Christum natum, Hieronymi Opera 15, 280-281: „Hilarius, cum apud Constantinopolim librum pro se Constantio porrexisset, ad Gallias redit".

${ }^{82}$ Contra Constantium Augustum, SCh 334, 166-223; anche PL 10, 578-604.
} 
Liber contra Constantium, ove il filoariano Costanzo viene presentato in modo pessimo: egli è peggiore dei più grandi persecutori dei cristiani! ${ }^{83}$

Anche la seguente costatazione esprime validamente, sebbene in un modo conciso e breve, la tragica e penosa situazione dei cattolici niceni durante l'impero di Costanzo:

„Quasi ogni Chiesa nel mondo è corrotta in nome della «pace» e con la complicità del capo degli ariani" $"$.

Anche la seguente costatazione geronimiana illustra bene la difficile situazione dei cattolici perseguitati dalla politica filoariana dell'imperatore. Di questi personaggi abbiamo già parlato, quindi non ripetiamo le notizie fornite in proposito.

Melezio di Sebaste, vescovo degli Armeni, viene trasferito ad Antiochia da Acacio e Giorgio, vescovi ariani, e dopo non molto spazio di tempo, ricevendo i presbiteri che erano stati sospesi [a divinis] dal suo predecessore Eudossio, per causa più che giusta, si comporta in modo falso cambiando fede ${ }^{85}$.

La notizia di san Girolamo, fornita qui sotto, loda l'operato di sant'Ilario in favore alla difesa della fede nicena; l'Atanasio occidentale non si piega alla pressione filoariana del potere secolare, ma respinge il sinodo di Rimini e difende la fede dei Trecentodiciotto Padri: „Grazie a Ilario, la Gallia condanna i pericolosi imbrogli di Rimini" ${ }^{\prime \prime 6}$. Ma ecco un'altra notizia di san Girolamo:

„Dopo la morte di Giorgio sul rogo, causata da una sommossa popolare, di quel Giorgio che era stato ordinato vescovo dagli ariani al posto di Atanasio, quest'ultimo torna ad Alessandria"87.

Giorgio di Cappadocia (o di Alessandria) fu vescovo ariano di Alessandria dal 357 al 24 dicembre 361. Una sollevazione popolare lo costrinse a fuggire a Costantinopoli per chiedere aiuto a Costanzo II, il quale diede a lui forze necessarie per domare la rivolta. Dal momento che Giorgio si era arricchito in modo illecito, sant'Atanasio narra che un decreto di deposizione fosse posto nei confronti di Giorgio dallo stesso concilio ariano di Seleucia. La morte di

${ }^{83}$ Cf. M. Simonetti, Ilario di Poitiers e la crisi ariana in Occidente, in: Quasten III 44.

${ }^{84}$ Hieronymus, «Chronicorum» Eusebii Caesariensis continuatio 284 Olymp., a. 359 post Christum natum, Hieronymi Opera 15, 280-281: „Omnes paene toto orbe ecclesiae sub nomine pacis et regis Arianorum consortio polluuntur".

${ }^{85}$ Cf. ibidem 284 Olymp., a. 360 post Christum natum, Hieronymi Opera 15, 280-281: „Meletius, Sebastiae Armeniorum episcopus, ab Acacio et Georgio episcopis Arianis Antiochiam transfertur et post non grande temporis intervallum cum presbyteros, qui ab Eudoxio antecessore suo depositi fuerant, suscepisset, exilii iustissimam causam subita fidei mutatione delusit".

${ }^{86}$ Ibidem 284 Olymp., a. 360 post Christum natum, Hieronymi Opera 15, 282-283: „Gallia per Hilarium Ariminensis perfidiae dolos damnat".

${ }^{87}$ Ibidem 285 Olymp., a. 362 post Christum natum, Hieronymi Opera 15, 282-283: „Georgio per seditionem populi incenso, qui in locum Athanasii ab Arianis fuerat ordinatus, Athanasius Alexandriam revertitur". 
Giorgio per linciaggio fu la conseguenza sia dei nuovi affronti alla popolazione di Alessandria, sia della morte del suo protettore Costanzo II, avvenuta il 3 novembre del $361^{88}$.

La notizia geronimiana che segue loda di nuovo Paolino di Antiochia presentandolo come il legittimo vescovo di quella città (ricordiamo che san Girolamo fu ordinato presbitero proprio dal luciferiano Paolino, capo della cosiddetta Piccola Chiesa di Antiochia):

„Eusebio [Sant'Eusebio di Vercelli] e Lucifero [San Lucifero di Cagliari] tornano dall'esilio. Dei due, Lucifero, unendosi a due altri confessori, ordina Paolino vescovo della parte cattolica di Antiochia, presbitero del vescovo Eustazio, e che non si era mai contaminato a causa della comunione con gli eretici" ${ }^{\prime 89}$.

Anche la notizia che segue denigra Melezio vedendo in lui uno che appoggia ,il dogma macedoniano":

„Si riunisce il sinodo di Antiochia, convocato da Melezio e dal suo gruppo. Dopo il rifiuto delle teorie della homoousia e dell'anomeismo, viene affermato il dogma macedoniano della homoiousia, a metà tra le due" ${ }^{90}$.

In realtà, questo sinodo (una ventina di vescovi di area siro-palestinese di ispirazione omea e omeousiana) si riunì, sotto la guida di Melezio, nel 363. I padri sinodali accettarono il Credo del I Concilio di Nicea del 325. Ciò nonostante, nella missiva inviata all'imperatore cattolico Gioviano diedero alla

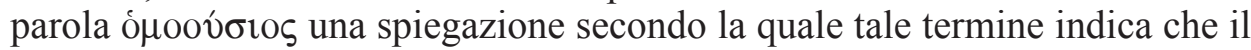

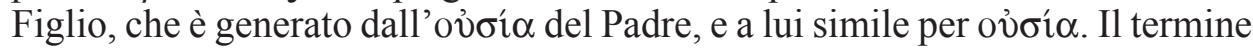

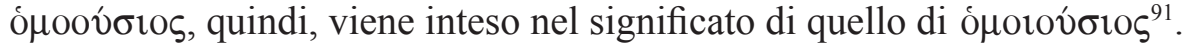

Anche la seguente notizia è collegata con l'eresia ariana o, meglio, pneumatomaca: „Valente, battezzato da Eudossio, vescovo degli ariani, ci perseguita" ${ }^{92}$.

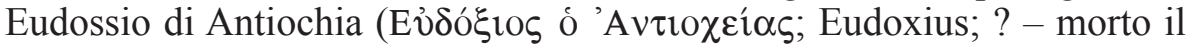
370), armeno di origine, fu vescovo ariano di Germanicia, di Antiochia e, infine, di Costantinopoli, dal 27 gennaio 360 alla sua morte. L'imperatore Valente si fece battezzare da Eudossio nel 367, in occasione della guerra contro i Goti. Nello stesso anno, Valente promulgò un ordine con il quale esiliava tutti quei

${ }^{88}$ Cf. M. Simonetti, Giorgio il Cappadoce, NDPAC II 2170.

${ }^{89}$ Hieronymus, «Chronicorum» Eusebii Caesariensis continuatio 285 Olymp., a. 362 post Christum natum, Hieronymi Opera 15, 282-283: „Eusebius et Lucifer de exilio regrediuntur, e quibus Lucifer adscitis duobus aliis confessoribus Paulinum, Eustathii episcopi presbyterum, qui se numquam haereticorum communione polluerat, in parte catholica Antiochiae episcopum facit”.

${ }^{90}$ Ibidem 285 Olymp., a. 364 post Christum natum, Hieronymi Opera 15, 286-287: „Synhodus Antiochiae a Melitio et suis facta, in qua homousio anomoeoque reiecto, medium inter haec homoeousion Macedonianum dogma vindicaverunt".

${ }^{91}$ Cf. M. Simonetti, Antiochia di Siria. II. Concili, NDPAC I 349-350.

${ }_{92}$ Hieronymus, "Chronicorum» Eusebii Caesariensis continuatio 286 Olymp., a. 366 post Christum natum, Hieronymi Opera 15, 288-289: „Valens ab Eudoxio Arianorum episcopo baptizatus nostros persequitur". 
vescovi ortodossi che erano stati esiliati da Costanzo e, in seguito, reintegrati da Giuliano l'Apostata ${ }^{93}$.

Dell'imperatore filoariano Valente san Girolamo fornisce anche questa notizia:

„Valente, obbligato a lasciare Antiochia, con un pentimento tardivo richiama la nostra gente dall'esilio"94.

Ancora una volta san Girolamo loda san Lucifero di Cagliari e degli altri vescovi cattolici intransigenti:

„Muore Lucifero, vescovo di Cagliari. Egli, come Gregorio [San Gregorio di Elvira ${ }^{95}$, vescovo delle province ispaniche, e Filone di Libia ${ }^{96}$, non fu mai coinvolto nella depravazione ariana"97.

9. Eunomiani e aeziani. Circa gli eunomiani e gli aeziani leggiamo: „A Costantinopoli compare Eunomio, discepolo di Aezio, da cui deriva l'eresia eunomiana"98.

Eunomio (Ev̉vó $\mu$ ıos; Eunomius; nato a Dacora in Cappadocia all'inizio del IV secolo, ? - morto nel 393 circa) fu un vescovo ariano, uno dei principali esponenti dell'arianesimo. Studiò teologia ad Alessandria sotto Aezio. Eudossio lo ordinò diacono e lo raccomandò come vescovo di Cizico nel $360^{99}$. Dopo un anno dalla nomina, Eudossio dovette sospendere Eunomio per volere di Costanzo II. Sotto gli imperatori Giuliano e Gioviano (360-364), Eunomio dimorò a Costantinopoli, stando a contatto con Aezio. Nel 365, l'usurpatore Procopio si fece acclamare imperatore: Eunomio si schierò con Procopio contro Valente. In seguito, Eunomio si stabilì nella propria villa a Calcedonia dando rifugio a Procopio (366). Nel 367 fu bandito in Mauretania ${ }^{100}$, ma venne

${ }^{93}$ Cf. Simonetti, Eudossio, NDPAC I 1823-1824.

${ }^{94}$ Hieronymus, "Chronicorum» Eusebii Caesariensis continuatio 289 Olymp., a. 378 post Christum natum, Hieronymi Opera 15, 302-303: „Valens de Antiochia exire conpulsus sera paenitentia nostros de exiliis revocat".

${ }^{95}$ Cf. F.J. Buckley, Christ and the Church according to Gregory of Elvira, Roma 1964; M. Simonetti, Gregorio di Elvira, NDPAC II 2459-2460.

${ }^{96}$ Cf. G.M. Pintus, La biografia di Lucifero di Cagliari nel "De viris illustribus" di Girolamo, in: Multas per gentes: Studi in memoria di Enzo Cadoni, a cura di Dipartimento di Scienze Umanistiche e dell'Antichità, Sassari 2000, 289-296.

${ }^{97}$ Hieronymus, «Chronicorum» Eusebii Caesariensis continuatio 287 Olymp., a. 370 post Christum natum, Hieronymi Opera 15, 292-293: „Lucifer Calaritanus episcopus moritur, qui cum Gregorio episcopo Hispaniarum et Philone Libyae numquam se Arianae miscuit pravitati".

${ }^{98}$ Ibidem 288 Olymp., a. 373 post Christum natum, Hieronymi Opera 15, 294-295: „Eunomius discipulus Aetii Constantinopoli agnoscitur, a quo haeresis Eunomiana".

${ }^{99}$ Cf. Philostorgius, HE III 20, ed. J. Bidez - F. Winkelmann, GCS 21, 2. Aufl., Berlin 1972 , 48, 19-23.

${ }^{100}$ Cf. R. Van Dam, Becoming Christian: The Conversion of Roman Cappadocia, Philadelphia 2003, 27. 
richiamato prima che arrivasse alla meta. Dopo la morte di Valente (378), tornò alle dispute religiose. Abitò in un sobborgo di Costantinopoli, celebrando in case private. Teodosio I esiliò Eunomio in una città nei pressi del Danubio, la quale fu però conquistata dai Goti ed Eunomio esiliato in Cappadocia ${ }^{101}$. Dopo la morte di Eunomio, Eutropio ordinò che il suo corpo fosse portato a Tiana e che le sue opere venissero bruciate ${ }^{102}$.

Aezio di Celesiria, fu noto anche come Aezio di Antiochia ('Ać $\tau$ ıs, Aetius; nato in Celesiria, ? - morto a Costantinopoli, nel 367). Lavorò come orefice ad Antiochia e si dedicò allo studio della filosofia e della medicina ${ }^{103}$. Nel $350 \mathrm{fu}$ ordinato diacono da Leonzio di Antiochia. Al primo sinodo di Sirmio, Aezio vinse un contrasto dialettico con i vescovi semiariani, Basilio di Ancira ed Eustazio di Sebaste, che cercarono allora di suscitare contro di lui Costanzo Gallo. Nel 356, Aezio si recò ad Alessandria con Eunomio per difendere l'arianesimo, ma venne bandito da Costanzo II. L'imperatore Giuliano lo richiamò dall'esilio e venne ordinato vescovo. Quando, nel 364, divenne imperatore Valente, si ritirò nella sua proprietà all'isola di Lesbo, per tornare a Costantinopoli, dove morì nel $367^{104}$.

Lapidale e piuttosto negativo è il giudizio di san Girolamo su Pietro II, vescovo di Alessandria, considerato comunemente ortodosso:

„Pietro viene ordinato $20^{\circ}$ vescovo di Alessandria. Dopo la morte di Valente, facilitò il rientro degli eretici, al punto che alcuni sospettavano che in cambio accettasse denaro" 105 .

Pietro II (?-381) fu vescovo di Alessandria, dal 373 alla sua morte. Nel 375 , vittima di una congiura ariana programmata dall'imperatore Valente e dal vescovo ariano di Antiochia, fu costretto a rifugiarsi a Roma. Fu sostituito dall'ariano alessandrino Lucio che, già dall'assassinio del vescovo alessandrino Giorgio, era considerato la guida degli ariani di Alessandria. Pietro fece ritorno ad Alessandria il 30 maggio 378, dopo la morte di Valente, e fu reinsediato al soglio vescovile grazie a un decreto del vescovo di Roma san Damaso

\footnotetext{
${ }^{101}$ Cf. ibidem, p. 37.
}

${ }^{102}$ Cf. Philostorgius, HE XI 5, GCS 21, 135, 22-27. Cf. anche F.W. Bautz, Eunomius, BBKL I 1558; R.P. Vaggione, Eunomius of Cyzicus and the Nicene Revolution, Oxford - New York 2000; J.L. Narvaja, Teologia y piedad en la obra de Eunomio de Cízico, Roma 2003; M. Simonetti, Eunomio di Cizico, NDPAC I 1836-1837.

${ }^{103}$ Cf. Philostorgius, HE III 15, GCS 21, 44, 8 - 47, 20.

${ }^{104}$ Cf. A. Jülicher, Aetios 6, RE I/1, 703; F.W. Bautz, Aetius, BBKL I 49-50; M. Simonetti, Aezio di Antiochia, NDPAC I 91-92.

${ }^{105}$ Hieronymus, «Chronicorum» Eusebii Caesariensis continuatio 288 Olymp., a. 373 post Christum natum, Hieronymi Opera 15, 296-297: „Alexandriae XX ordinatur episcopus Petrus, qui post Valentis interitum tam facilis in recipiendis haereticis fuit, ut nonnullis suspicionem acceptae pecuniae intulerit". 
I che l'aveva ospitato. Pietro morì due anni dopo. È citato nell'editto di Tessalonica, del 380, promulgato da Teodosio $\mathrm{I}^{106}$.

L'ultima notizia riguardante l'arianesimo e, precisamente l'ariano Aussenzio di Milano, viene data da san Girolamo quasi obiter, mentre parla dell'ordinazione episcopale di sant'Ambrogio:

„Dopo la tardiva morte di Aussenzio, a Milano Ambrogio viene ordinato vescovo e ognuno in Italia viene convertito alla giusta fede" 107 .

Aussenzio di Milano (nato probabilmente a Durostorum [Silistra] in una data per noi sconosciuta - morto a Milano, nel 374) fu vescovo ariano di Milano dal 355 fino alla sua morte. Era originario della Cappadocia, o era Goto, proveniente dalla città di Durostorum, l'odierna Silistra, sul delta del Danubio. Nel 343, Aussenzio fu ordinato diacono ad Alessandria dall'ariano Gregorio di Cappadocia. In seguito andò a Milano, diventando discepolo di Dionisio, vescovo di quella città. Quando Costanzo II depose Dionisio, in quanto cattolico, Aussenzio, appoggiato dall'imperatrice filoariana Giustina, fu eletto vescovo di Milano. Nel 369, Aussenzio fu scomunicato dal vescovo di Roma, san Damaso. Aussenzio rimase vescovo di Milano fino alla sua morte, allorché venne quindi sostituito da sant'Ambrogio ${ }^{108}$.

Aurelio Ambrogio (Aurelius Ambrosius; nato a Treviri, nel circa 339340 - morto a Milano, nel 397): il suo biografo, Paolino, narra nella Vita S. Ambrosii 7-8 che, allo scopo di dissuadere i milanesi dall'eleggerlo vescovo, sant'Ambrogio provò anche a macchiare la sua buona fama. Ma, dato che i fedeli non demordevano, tentò addirittura di fuggire. I milanesi, quindi, si rivolsero all'imperatore Valentiniano I. Allora, sant'Ambrogio fu ordinato vescovo, probabilmente il 7 dicembre $374^{109}$.

10. Audiani. San Girolamo, nella sua Cronaca, menziona Audio e i suoi seguaci, gli audiani, soltanto una sola volta:

${ }^{106} \mathrm{Cf}$. Александрийская Православия Церковь, in: Православная Энииклопедия, I, Москва 2000, 559-594; M. Simonetti, Pietro II di Alessandria, NDPAC III 4077-4078.

107 Hieronymus, «Chronicorum» Eusebii Caesariensis continuatio 288 Olymp., a. 374 post Christum natum, Hieronymi Opera 15, 298-299: „Post Auxentii seram mortem Mediolani, Ambrosio episcopo constituto, omnis ad fidem rectam Italia convertitur".

${ }^{108}$ Cf. Athanasius Alexandrinus, Historia Arianorum ad monachos 75, PG 25, 784B-C; D.H. Williams, The Anti-Arian Campaigns of Hilary of Poitiers and the "Liber contra Auxentium", ChH 61 (1992) 7-22; M. Simonetti, Aussenzio di Milano, NDPAC I 662-663.

${ }^{109}$ Cf. F. Homes Dudden, The Life and Times of St. Ambrose, Oxford 1935; A. Paredi - J. Costelloe, Saint Ambrose: His Life and Times, Notre Dame, IN 1964; N.B. McLynn, Ambrose of Milan: Church and Court in a Christian Capital, The Transformation of the Classical Heritage 22, Berkeley 1994; Ch. Markschies, Ambrosius von Mailand und die Trinitätstheologie, Tübingen 1995; C. Pasini, Ambrogio di Milano. Azione e pensiero di un vescovo, Cinisello Balsamo 1996; E. Dassmann, Autobiographie in Hagiographie. Beobachtungen zu den Mönchsviten und einigen Nekrologen des Hieronymus, „Anuario de Historia de la Iglesia” 8 (1999) 109-124; M.G. Mara, Ambrogio di Milano, NDPAC I 229-235. 
„Audio acquista importanza in Celesiria ${ }^{110}$, da lui prende nome l'eresia audiana"111.

Questa setta ascetica fu fondata da Audio (Audeus o Audius), arcidiacono di Edessa, per i suoi dissidi con la Chiesa ufficiale sul calcolo della data della Pasqua e per la critica della ricchezza e la dissolutezza di alcuni vescovi. Audio fu esiliato nell'odierna Ucraina e iniziò un'opera di conversione dei Goti $^{112}$. San Girolamo scrive contro Audio anche altrove ${ }^{113}$.

Sant'Epifanio di Salamina, che considerava gli Audiani antropomorfiti, scrive che essi accusavano la Chiesa di aver cambiato la data della Pasqua per ubbidire al volere dell'imperatore Costantino $\mathrm{I}^{114}$.

Teodoreto di Cirro, infine, li presenta come gnostici e manichei influenzati dall'Apocalisse di Abramo ${ }^{115}$.

11. Fotiniani. Anche Fotino e i suoi seguaci vengono menzionati dalla Cronaca di san Girolamo soltanto un'unica volta. Il Dalmata parla solo della morte dell'eresiarca, avvenuta in Galazia nell'anno 376, e scorge nella dottrina di Fotino degli influssi di stampo giudaizzante:

„Fotino, che aveva dato inizio all'insegnamento giudaizzante dei fotiniani, muore in Galazia"116.

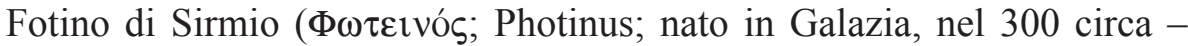
morto in Galazia, nel 376) fu vescovo di Sirmio (città della Pannonia, residenza di Costanzo II). Fu diacono e discepolo di Marcello, metropolita di Ancira

${ }^{110}$ La Celesiria ( $\dot{\eta} \kappa o i ́ \lambda \eta \Sigma v$ pí $\alpha$ - „la Siria concava”, in lat. Syria Coele) era il nome dato dai Greci, e adottato dai Romani quando fecero di essa una loro provincia, che si estendeva dalle catene del Monte Libano e dell'Anti-Libano fino alla pianura della Beqā' $\bar{a}^{-}$Circa la Celesiria, cf. O. Keel - M. Küchler - Ch. Uehlinger, Orte und Landschaften der Bibel. Ein Handbuch und Studien-Reiseführer zum Heiligen Land, I: Geographisch-geschichtliche Landeskunde, Göttingen 1984, 237-239.

111 Hieronymus, "Chronicorum» Eusebii Caesariensis continuatio 280 Olymp., a. 341 post Christum natum, Hieronymi Opera 15, 260-261: „Audeus in Syria Coele clarus habetur, a quo haeresis Audiana".

${ }^{112}$ Cf. A.A. Vasiliev, The Goths in the Crimea, Cambridge MA 1936, passim; H. Wolfram, Die Goten, München 1990, passim; B. Degórski, Hieronymi Opera 15, 260.

${ }^{113} \mathrm{Cf}$. ad esempio, Hieronymus, Epistula VI 1-2, ed. I. Hilberg, CSEL 54, Vindobonae - Lipsiae 1910, 24, $1-25,17$.

${ }^{114}$ Cf. Epiphanius Constantiensis, Panarion 70, 10, 3-5, ed. K. Holl - J. Dummer, GCS 37, Berlin 1985, 243, 9-18.

${ }^{115}$ Cf. Theodoretus Cyrrhenus, HE IV 10, 1-4, ed. P. Canivet, SCh 530, Paris 2009, 218-220. Cf. anche Epiphanius Constantiensis, Panarion 70, GCS 37, 232, 16 - 249, 8; Socrates, HE VI 7, 27, GCS NF 1, 324, 19. Circa gli Audiani, cf. T. Orlandi, Audiani, NDPAC I 650.

${ }^{116}$ Hieronymus, "Chronicorum» Eusebii Caesariensis continuatio 288 Olymp., a. 376 post Christum natum, Hieronymi Opera 15, 300-301: „Fotinus in Galatia moritur, a quo Fotinianorum dogma Iudaicum". 
e, anch'egli, galata ${ }^{117}$. Entrambi si contrapposero all'eresia degli ariani. Fotino fu condannato sia dagli ariani, nel 344 dal sinodo ariano di Antiochia, sia dagli ortodossi, che lo riconobbero colpevole nei due sinodi filoariani di Milano (345 e 347) e nel sinodo di Sirmio del 351, in cui venne deposto con l'accusa di sabellianismo e adozionismo ${ }^{118}$. La condanna definitiva di Fotino e dei suoi seguaci fu emessa dal primo Concilio di Costantinopoli del 381, e confermata dal vescovo di Roma san Damaso I, nel 382. Tuttavia, sembra che Fotino sia ritornato alla sede episcopale durante il regno dell'imperatore Giuliano l'Apostata (361-363), per poi essere definitivamente bandito dall'imperatore Valentiniano I, nel $364^{119}$.

\section{$* * *$}

Girolamo, traducendo l'opera di Eusebio di Cesarea, intende essere fedele e rigoroso nelle informazioni su persone e fatti. Tuttavia, quando affronta eretici e loro sostenitori, imperatori o vescovi che siano, ed eresie, non risparmia tra le righe il giudizio che il suo impulso gli detta. In ogni caso, dalle informazioni che tramanda e che sono per lo più di storia a lui contemporanea, trapela un complesso quadro geopolitico che nel sec. IV influenzò la composizione del dogma della Grande Chiesa con lotte intestine e travaglio teologico, fino all'affermazione della verità. Interessi di potere fuori e dentro la gerarchia ecclesiastica, con la complicità degli imperatori pro o contro i legittimi detentori delle varie sedi episcopali, provocarono maggior approfondimento del dato di fede, ma anche richiesero l'eroicità indefessa di molti e santi testimoni e confessori della stessa.

Girolamo non trascura i Concili e i Sinodi del sec. IV e quanto avvenne dietro le quinte di queste significative assemblee, la manipolazione o meno a cui andarono soggette, i loro protagonisti tra ortodossi ed eterodossi, occidentali ed orientali. All'elenco di eretici ed eresie nel Chronicon trovano spazio anche frange e propaggini che, perifericamente alla principali diatribe, riemergevano e che non sfuggono all'attenzione del Dalmata. Nella propria vita egli dedicò più opere in risposta a eretici come Elvidio e Gioviniano ed ebbe in orrore l'arianesimo ancora all'alba del sec. V quando i barbari di Alarico commisero il sacco di Roma.

Dunque, è ovvio che Girolamo non può risparmiare allora giudizi e annotazioni attente anche a particolari che forse a noi, distanti di secoli, possono

${ }^{117}$ Cf. Socrates, HE II 18, 7, GCS NF 1, 112, 7-9; II 19, 16, GCS NF 1, 114, 25 - 115, 1.

${ }^{118}$ Cf. ibidem II 29, 1-5, GCS NF 1, 140, 9 - 141, 5; Sozomenus, HE IV 6, 1-16, GCS 50, $143,13-146,8$.

${ }^{119}$ Circa Fotino cf. K.H. Uthemann, Photeinos von Sirmium, LThK VIII 267; D.H. Williams, Monarchianism and Photinus of Sirmium as the Persistent Heretical Face of the Fourth Century, HTR 99 (2006) 167-206; M. Simonetti, Fotino di Sirmio, NDPAC II 1998-1999; Degórski, Hieronymi Opera 15 , p. 300. 
sfuggire, oppure contraddire opinioni di storici qualificati dall'analisi di più ampio criterio. San Girolamo fu un osservatore ,coinvolto” con passione nelle vicende del suo tempo. Questo fa di lui non solo un traduttore, bensì pure un acceso cronista di quei fatti e di quei protagonisti che vuole tramandare da testimone della verità non interessato alla lotta politica. Notizie di una lotta per il dogma nel quale spese la propria vita con genuino ardore lo vedono all'opera, quindi, in modo non asettico, con l'investimento del suo arguto genio a cui nulla sfugge e che non ricusa, tra le righe, la propria opinione pur di condurre i lettori e i posteri a nulla cedere e concedere là dove il compromesso di uno „iota” induce all'eclisse della verità.

\section{HERETICS AND HERESIES IN THE CHRONICON OF ST. JEROME}

\section{(Summary)}

In the translation of the Chronicon of Eusebius of Caesarea, St. Jerome firmly lists all the heretics and the heresies which - with the complicity of struggles for political power - affected Christianity, causing a sort of internal „martyrdom” for those defending faith. Donatists, Arianists, Macedonians, Anomoeans, Aetians, Audians, Photinians: none escapes the translation and judgement of the Great Dalmatian, who sometimes - impelled by the concise writing style of a chronicle expresses his own judgement on heretics and their supporters with a single harsh qualification, failing to provide wider evaluations. The fights for the truth invested even the Synods of Rimini and Seleucia, but his concise and keen exposition of the events provides to the reader the information on the facts as well as on the political interference of many decisions, which were not prompted by religious motivations. Once again, an overview of the events presented in his Chronicon - through his ,translation” and "tradition” - confirms how divisions and problems within the Church in the past centuries were caused by external motives, as well as often spoiled by the political events and took advantage of the frailty of the human nature, rather than aiming at seeking the truth.

\section{HERETYCY I HEREZJE W CHRONICON ŚW. HIERONIMA}

\section{(Streszczenie)}

Kontynuując Chronicon Euzebiusza z Cezarei, wśród wielu innych postaci św. Hieronim wymienia także błędnowierców i odszczepieńców IV w. Wskazuje przy tym częstokroć na ich związki z walką polityczną i władzą świecką, która w owym czasie nękała prawowiernych chrześcijan, czyniąc z nich swoistych „męczenników". Donatyści, arianie, macedonianie (duchobórcy), eunomianie, aecjanie, audianie, fotynianie nie uniknęli surowej oceny wielkiego Ojca Kościoła, 
który niekiedy - ograniczony swoistym, krótkim stylem samej Kroniki - nawet poprzez jeden jedyny przymiotnik wyraża całą swoją krytykę i dezaprobatę względem błędnowierców oraz tych, którzy ich wspierali i im schlebiali. Nieustanne walki o czystość wiary nie zawsze kończyły się zwycięstwem, jak to widać np. w postanowieniach synodów z Rimini i Seleucji. Niemniej jednak przekaz faktów, dokonany przez Hieronima, pozwala czytelnikowi poznać choćby w zarysie przebieg i cały niesprzyjający kontekst polityczny tych teologicznych sporów. Po raz kolejny lektura faktów i ich kontekstu historyczno-politycznego potwierdza, iż rozłamy i trudności wewnątrz Kościoła pierwszych wieków były spowodowane działaniami zewnętrznymi władz świeckich, wykorzystującymi ludzką słabość spragnioną raczej władzy niż prawdy.

Key words: St. Jerome, Eusebius of Caesarea, Ancient Church History ( $4^{\text {th }}$ century), Heresies, patristic Theology, Donatism, Arianism, Macedonians, Council of Rimini, Council of Seleucia, Pneumatology, Aëtians and Eunomians, Audians, Photinians.

Parole chiave: san Girolamo, Eusebio di Cesarea, storia della Chiesa antica (IV sec.), eresie, teologia patristica, donatisti, ariani, macedoniani, i sinodi di Rimini e di Seleucia, pneumatologia, eunomiani e aeziani, audiani, fotiniani.

Słowa kluczowe: św. Hieronim, Euzebiusz z Cezarei, historia Kościoła starożytnego (IV w.), herezje, teologia patrystyczna, donatyści, arianie, macedonianie, synody w Rimini i Seleucji, pneumatologia, eunomianie i aecjanie, audianie, fotynianie.

\title{
BIBLIOGRAFIA
}

\author{
Fonti \\ 1. Fonti principali
}

le edizioni del Chronicon di Eusebio di Cesarea

e della sua continuazione fatta da san Girolamo

Eusebius CAesariensis, Chronicon bipartitum, nunc primum ex Armeniaco textu in Latinum conversum, adnotationibus auctum, Graecis fragmentis exornatum, ed. J.B. Aucher, Venetiis 1818.

Eusebius Caesariensis, Chronicon id est Temporum Breviarium, ed. Ratdolt, Venetiis 1483.

Eusebius Caesariensis, Chronicorum libri duo, ed. A. Schoene, Berolini 1866-1875.

Eusebius Caesariensis, Werke, Bd. 5: Die Chronik, aus dem Armenischen übersetzt, hrsg. von J. Karst, GCS 20, Berlin 1911.

Eusebius Caesariensis, Werke, Bd. 7: Die Chronik des Hieronymus, hrsg. von R. Helm, 2. Aufl., GCS 47, Berlin 1956.

[Hieronymus], Opere di Girolamo, vol. 15: Opere storiche e agiografiche, a cura di B. Degórski, Roma 2014. 


\section{Fonti secondarie}

Agapius Hierapolitanus, Historia universalis, ed. B. Pirone: Agapio di Gerapoli Storia universale, introduzione, traduzione dall'arabo e note di B. Pirone, Studia Orientalia Christiana. Monographiae 21, Milano 2013.

Ammianus Marcellinus, Res gestae. Libri qui supersunt, ed. W. Seyfarth, I-II, Leipzig 1978.

Athanasius AleXandrinus, Historia arianorum ad monachos, PG 25, 695-796.

Cassiodorus, Chronica, PL 69, 1213-1247; ed. T. Mommsen, MGH Auctores Antiquissimi XI, Berolini 1894, 109-161.

Epiphanius Constantiensis, Panarion, ed. K. Holl - J. Dummer, GCS 37, Berlin 1985.

Gesta inter Liberium et Felicem episcopos, ed. O. Guenther, in: Collectio Avellana, CSEL 35/1, Pragae - Vindobonae - Lipsiae 1895, 1-4.

Hieronymus, Epistulae, pars I: Epistulae I-LXX, ed. I. Hilberg, CSEL 54, Vindobonae Lipsiae 1910.

Hilarius Pictaviensis, Apologetica ad reprehensores libri De synodis responsa, PL 10, 545-548.

Hilarius Pictaviensis, Tractatus super Psalmos, ed. J. Doignon, SCh 515, Paris 2008; ed. J. Doignon - R. Demeulenaere, CCL 61-61A-B, Turnhout 1997-2009.

Hilarius Pictaviensis, Contra Arianos vel contra Auxentium Mediolanensem, PL 10, 609-618.

Hilarius Pictaviensis, Contra Constantium Augustum, PL 10, 578-604; ed. A. Rocher, SCh 334, Paris 1987.

Hilarius Pictaviensis, De mysteriis, ed. A.L. Feder in: Hilarius Pictaviensis, Opera, CSEL 65, Vindobonae 1916, 1-38; PLS 1, 246-270; ed. J.-P. Brisson, SCh 19bis, Paris 1967.

Hilarius Pictaviensis, De synodis seu de fide orientalium, PL 10, 471-546.

Hilarius Pictaviensis, Fragmenta historica, ed. A.L. Feder in: Hilarius Pictaviensis, Opera, CSEL 65, Vindobonae 1916, 39-193; PL 10, 627-724.

Hilarius Pictaviensis, Hymni, ed. A.L. Feder in: Hilarius Pictaviensis, Opera, CSEL 65, Vindobonae 1916, 207-223; PLS 1, 271-281.

Hilarius Pictaviensis, Commentarius in Evangelium Matthaei, PL 9, 917-1076; ed. J. Doignon, t. 1-2, SCh 254 e 258, Paris 1978-2007; ed. A.L. Feder in: Hilarius Pictaviensis, Opera, CSEL 65, Vindobonae 1916, 232 [frammenti]).

Hilarius Pictaviensis, De Trinitate, ed. P. Smulders, t. 1-3, SCh 443, 448, 462, Paris 1999-2000-2001.

Libanius, Opera, ed. R. Foehrster, I-IV, Lipsiae 1903-1908.

Philostorgius, Historia ecclesiastica, ed. J. Bidez - F. Winkelmann, GCS 21, 2. Aufl., Berlin 1972.

Prosper Aquitanus, Epitoma Chronicae, PL 51, 535-606; ed. T. Mommsen, MGH Auctores Antiquissimi IX, Berolini 1892, 385-485; continuazione, ivi, 486-499; additamen$t a$, PLS 3, 147-148).

Rufinus Aquileiensis, Historia Ecclesiastica, PL 21, 463-540.

Socrates, Historia ecclesiastica, ed. G.Ch. Hansen, GCS NF 1, Berlin 1995.

Sozomenus, Historia ecclesiastica, ed. J. Bidez - G.Ch. Hansen, GCS 50, Berlin 1960.

Theodoretus Cyrrhenus, Historia ecclesiastica, ed. P. Canivet, t. 2: Livres III-IV, SCh 530, Paris 2009.

Victor Tunnunensis, Chronica, PL 68, 941-962; ed. T. Mommsen, MGH Auctores Antiquissimi XI, Berolini 1894, 163-206; ed. C. Cardelle de Hartmann, CCL 173A, Turnhout 2002. 


\section{Studi}

Александрийская Православная Церковь, in: Православная Энииклопедия, I, Москва 2000, 559-594.

BAutz F.W., Aetius, BBKL I 49-50.

BAUTz F.W., Eunomius, BBKL I 1558.

BAUTz F.W., Eusebius von Vercelli, BBKL I 1568-1569.

Boardman J., The Cambridge Ancient History, Cambridge 1970.

Buckley F.J., Christ and the Church according to Gregory of Elvira, Roma 1964.

CAmplani A., Atanasio di Alessandria, NDPAC I 622.

CoRti G., Lucifero di Cagliari. Una voce nel conflitto tra chiesa e impero alla metà del IV secolo, SPM 24, Milano 2004.

Crouzel H. - Odrobina L., Rodanio di Tolosa, NDPAC III 4573-4574.

Cuneo P.O., La legislazione di Costantino II, Costanzo II e Costante (337-361), Milano 1997.

Dalvit M., Montenses. The Ecclesiological Reflection of the Donatists Based on the Habacuc's Prophecy, „Augustiniana” 63 (2013) 173-188.

Dassmann E., Autobiographie in Hagiographie. Beobachtungen zu den Mönchsviten und einigen Nekrologen des Hieronymus, „Anuario de Historia de la Iglesia” 8 (1999) 109-124.

DATTRINO L., Eusebio di Vercelli, NDPAC I 1861-1862.

Dieu Trinité d'hier à demain avec Hilaire de Poitiers, Actes du congrès-colloque du Futuroscope de Poitiers (15-17 novembre 2002), ed. D. Bertrand, Paris 2010.

EVEREtT N., Narrating the Life of Eusebius of Vercelli, in: Narrative and History in the Early Medieval West, ed. R. Balzaretti - E.M. Tyler, Turnhout 2006, 133-165.

Forlin PATrucco M., Costanzo II, NDPAC I 1247-1248.

Gigli G., L'ortodossia, l'arianesimo e la politica di Costanzo II, Napoli 1949.

Grisart A., La Chronique de Saint Jérôme. Le lieu et la date de sa composition, „Hélikon” 2 (1962) 248-258.

Homes Dudden F., The Life and Times of St. Ambrose, Oxford 1935.

Jones A.H.M., The Career of Flavius Philippus, „Historia” 4 (1955) 229-233.

The Prosopography of the Later Roman Empire, ed. A.H.M. Jones - J.R. Martindale - J. Morris, III, Cambridge 1992.

Jülicher A., Aetios 6, RE I/1, 70.

JÜLICHER A., Eusebios 27, RE VI/1, 1441-1443.

KANNENGIESSER CH., Eusebio di Nicomedia, NDPAC I 1857-1860.

Keel O. - Küchler M. - Uehlinger CH., Orte und Landschaften der Bibel. Ein Handbuch und Studien-Reiseführer zum Heiligen Land, I: Geographisch-geschichtliche Landeskunde, Göttingen 1984.

Krüger S.G., Eusebius, Bischof von Emesa (Emisa) in Phönizien, in: Realencyklopädie für protestantische Theologie und Kirche, V, Leipzig 1898, 618-619.

LaConi S., Costanzo II. Ritratto di un imperatore eretico, Roma 2004.

Leroux J.-M., Acace, évêque de Césarée de Palestine (341-365), StPatr 8 (1966) 82-85.

LiETzManN H., Hilarius 11, RE VIII/2, 1601-1604.

Mara M.G, Ambrogio di Milano, NDPAC I 229-235.

Markschies Ch., Ambrosius von Mailand und die Trinitätstheologie, Tübingen 1995.

Marone P., Felice II antipapa, NDPAC II 1927.

McLynn N.B., Ambrose of Milan: Church and Court in a Christian Capital, The Transformation of the Classical Heritage 22, Berkeley 1994.

Mondin B., Dizionario dei Teologi, Bologna 1992. 
Narvaja J.L., Teologia y piedad en la obra de Eunomio de Cízico, Roma 2003.

Neyses A., Die Baugeschichte der ehemaligen Reichsabtei St. Maximin bei Trier, I-II, Trier 2001.

Orlandi T., Audiani, NDPAC I 650.

Орловъ А., Тринитарныя воззрбнія Иларія Пиктавійскаго: историко-догматическое изсльдованіе, Сергіевъ Посадъ 1908.

Орловъ А., Христологія Иларія Пиктавійскаго: въ связи съ обзоромъ христологическихъ ученій 2-4 вв., Сергіевъ Посадъ 1909.

Paredi A. - Costelloe J., Saint Ambrose: His Life and Times, Notre Dame 1964.

Pasini C., Ambrogio di Milano. Azione e pensiero di un vescovo, Cinisello Balsamo 1996.

Pintus G.M., La biografia di Lucifero di Cagliari nel "De viris illustribus" di Girolamo, in: Multas per gentes: Studi in memoria di Enzo Cadoni, a cura di Dipartimento di Scienze Umanistiche e dell'Antichità, Sassari 2000, 289-296.

Pohlsander H.A., Maximinus und Paulinus. Zwei Bischöfe im vierten Jahrhundert, „Trierer Zeitschrift" 59 (1996) 157-160.

Pollastri A., Eufronio di Antiochia, NDPAC I 1826.

SAMUlowitz S., Vitale di Antiochia, NDPAC III 5664-5665.

SAUGET J.-M., Massimo, NDPAC II 3116-3118.

SAuser E., Lucifer, Bischof von Calaris, BBKL V 298-301.

SAXer V. - HeId S., Massimino di Treviri, NDPAC II 3116.

SaXer V. - SAmulowitz S., Saturnino di Arles, NDPAC III 4768-4769.

Scorza Barcellona F., Vittore di Tunnuna, NDPAC III 5678-5679.

Simonetti M., Acacio di Cesarea, NDPAC I 36-37.

Simonetti M., Aezio di Antiochia, NDPAC I 91-92.

Simonetti M., Antiochia di Siria. II. Concili, NDPAC I 349-350.

Simonetti M., Cirillo di Gerusalemme, NDPAC I 1050-1052.

Simonetti M., Dionigi di Milano, NDPAC I 1443.

Simonetti M., Doroteo di Antiochia, NDPAC I 1508.

Simonetti M., Eudossio, NDPAC I 1823-1824.

Simonetti M., Eulalio di Antiochia, NDPAC I 1833.

Simonetti M., Eunomio di Cizico, NDPAC I 1836-1837.

Simonetti M., Eusebio di Emesa, NDPAC I 1854-1855.

Simonetti M., Eustazio di Antiochia, NDPAC I 1862-1863.

Simonetti M., Euzoio di Antiochia, NDPAC I 1876-1877.

Simonetti M., Filogonio di Antiochia, NDPAC II 1958.

Simonetti M., Fotino di Sirmio, NDPAC II 1998-1999.

Simonetti M., Gregorio di Elvira, NDPAC II 2459-2460.

Simonetti M., Giorgio il Cappadoce, NDPAC II 2170.

Simonetti M., Ilario di Poitiers, NDPAC III 2521-2528.

Simonetti M., Leonzio di Antiochia, NDPAC II 2776.

Simonetti M., Lucifero (luciferiani), NDPAC II 2935-2937.

Simonetti M., Macedonio (macedoniani), NDPAC II 2961-2962.

Simonetti M., Melezio di Antiochia, NDPAC II 3190-3191.

Simonetti M., Paolino di Tiro, NDPAC III 3832.

Simonetti M., Paolino di Treviri, NDPAC III 3832-3833.

Simonetti M., Paolo di Costantinopoli, NDPAC III 3852-3853.

Simonetti M., Pietro II di Alessandria, NDPAC III 4077-4078.

Simonetti M., Rimini (concilio), NDPAC III 4509-4511.

Simonetti M., Seleucia d'Isauria (concilio), NDPAC III 4843-4844. 
Smulders P., La Doctrine trinitaire de St. Hilaire de Poitiers. Esquisse du mouvement dogmatique depuis le Concile de Nicée jusqu'au règne de Julien, Analecta Gregoriana 32, Roma 1944.

Starowieyski M., Cirillo di Gerusalemme (Pseudo), NDPAC I 1052.

STUdER B., Liberio papa, NDPAC II 2822-2823.

Swift L.J. - James H.O., Constantius II on Flavius Philippus, „The American Journal of Philology" 83 (1962) 247-264.

Uthemann K.H., Photeinos von Sirmium, LThK VIII 267.

Vaggione R.P., Eunomius of Cyzicus and the Nicene Revolution, Oxford - New York 2000.

DAM R. VAN, Becoming Christian: The Conversion of Roman Cappadocia, Philadelphia 2003.

VAsiliev A.A., The Goths in the Crimea, Cambridge MA 1936.

VoICU S.J., Macario I, NDPAC II 2953.

Williams D.H., The Anti-Arian Campaigns of Hilary of Poitiers and the "Liber contra Auxentium", ChH 61 (1992) 7-22.

Williams D.H., Monarchianism and Photinus of Sirmium as the Persistent Heretical Face of the Fourth Century, HTR 99 (2006) 167-206.

Wolfram H., Die Goten, München 1990.

Zöckler O., Macarius, in: New Schaff-Herzog Encyclopedia of Religious Knowledge, ed.

S.M. Jackson, VII, London - New York $1914^{3}, 103$. 